\title{
A Novel Angular Texture Pattern (ATP) Extraction Method for Crop and Weed Discrimination Using Curvelet Transformation
}

\author{
P Prema* and D Murugan $^{+}$ \\ *Agricultural College and Research Institute, Madurai-625104, Tamil Nadu, India. \\ ${ }^{+}$Manonmaniam Sundaranar University, Tirunelveli, Tamil Nadu, India.
}

Received 2nd Nov 2015; accepted 22nd May 2016

\begin{abstract}
Weed management is the most significant process in the agricultural applications to improve the crop productivity rate and reduce the herbicide application cost. Existing weed detection techniques does not yield better performance due to the complex background and illumination variation. Hence, there arises a need for the development of effective weed identification technique. To overcome this drawback, this paper proposes a novel Angular Texture Pattern (ATP) Extraction Method for crop and weed discrimination using curvelet transformation. In our proposed work, Adaptive Median Filter (AMF) is used for filtering the impulse noise from the image. Plant image identification is performed using green pixel extraction and K-means clustering. Wrapping based Curvelet transform is applied to the plant image. Feature extraction is performed to extract the angular texture pattern of the plant image. Particle Swarm Optimization (PSO)-based Differential Evolution Feature Selection (DEFS) approach is applied to select the optimal features. Then, the selected features are learned and passed through an RVM-based classifier to find out the weed. Edge detection and contouring is performed to identify the weed in the plant image. The fuzzy rule-based approach is applied to detect the low, medium and high levels of the weed patchiness. From the experimental results, it is clearly observed that the accuracy of the proposed approach is higher than the existing Support Vector Machine (SVM) based approaches. The proposed approach achieves higher accuracy, sensitivity, specificity, Hausdorff distance, Jaccard distance and Dice distance.
\end{abstract}

Key words: Angular Texture Pattern (ATP) extraction method, Adaptive Median Filter (AMF), Convoluted Gray Level Co-occurrence Matrix (CGLCM), Curvelet Transformation, Mobility Window Projection (MWP), Weed Identification

\section{Introduction}

Weeds are the undesirable plants that can reproduce in the agricultural field and disturb the overall crop yield. Uncontrolled weeds can reduce the crop yield from $10 \%$ to about $90 \%$. Hence, weed control is considered as a vital practice in the agricultural systems, to sustain the crop productivity and quality. In most cases, weed control depends on the usage of chemical herbicides to achieve effective control of weed infestations and obtain high profit. But, the application of chemical herbicides causes adverse effects on the environment and requires more manual labor and expense. If the same types of herbicides are repeatedly applied in the field for the removal of the weeds, there is often a chance of the emergence of weeds to become tolerant to those types of herbicides. This leads to the increasing interest in the development of alternative weed control techniques. To reduce the burden

\footnotetext{
Correspondence to: pp76@tnau.ac.in

Recommended for acceptance by Carlo Sansone

http://dx.doi.org/10.5565/rev/elcvia.826

ELCVIA ISSN: $1577-5097$

Published by Computer Vision Center / Universitat Autonoma de Barcelona, Barcelona, Spain
} 
on the agricultural sector, the crop yield should be increased with reduced cost for weed control. Conventionally, a machine vision system can distinguish the crops from the weeds for the effective application of the herbicides and improvement in the crop yield with reduced environmental degradation. An automated system is used in this approach for acquiring the image of different areas of the crop field. Previously, classification of the crops and weeds is performed based on the geometrical characteristics such as leaf shape or plant structure and spectral reflectance characteristics. The ground-based sensor technologies are used to identify the weeds. The development of the computer vision capabilities enables reliable and rapid identification of weeds [1]. Detection of weed using color or shape characteristics is simple and efficient, but the adaptability of this technique is very poor. The weed recognition method using multispectral characteristics is feasible. However, it is very expensive.

Several existing approaches address the problem of green identification under the assumption that plants show a high level of greenness, but the loss of the greenness due to various reasons is not considered. Consequently, the ability to develop reliable and accurate techniques for the weed identification is regarded as a high priority for the effective agriculture management. To overcome the limitations of the existing weed identification techniques, this paper proposes a novel ATP extraction method for crop and weed discrimination using curvelet transformation. AMF filters the impulse noise from the image. Green pixel extraction is performed to obtain the green pixel count from the filtered image. K-means clustering is applied to the filtered image, to cluster the soil and plants. Then, the green pixel count is verified with the pixel count of the clusters. If the green pixel count is nearest to the pixel count of the clusters, then it is considered as the plant image. Wrapping based curvelet transformation is applied to the plant image. After applying the curvelet transformation, the angular based features are extracted from the transformed image. Feature extraction is performed to extract the angular texture pattern of the plant image. A Mobility Window Projection (MWP) system represents the movement of the window to each patch of the image. In the texture pattern analysis, the MWP system is used to analyze the variation in the texture. The Tamura features are extracted from the image, Gabor filter with four orientations is applied to the image and GLCM features are extracted from the Gabor filtered image. PSO-based DEFS approach is applied to select the optimal features. Then, the selected features are learned and passed through an RVM-based classifier to find out the weed. Edge detection and contouring is performed for the identification of weed in the plant image. The low, medium and high levels of the weed patchiness are detected using the Fuzzy rule-based approach. From the experimental results, it is clearly observed that the performance of the proposed approach is higher than the existing SVM-based approaches.

The rest of the paper is organized as follows: Section II describes an overview of the existing approaches related to the identification of weeds. Section III explains the proposed novel ATP extraction method including the AMF, plant image identification, curvelet transformation, feature extraction, PSO-based DEFS, RVM-based classification, weed edge detection and contouring and Fuzzy-rule based weed patchiness processes. Section IV presents the performance analysis results of the proposed method. Section V illustrates the conclusion and future scope of the proposed work.

\section{Related Work}

In the conventional cropping systems, weed was removed by applying chemical herbicides on the plants. However, this practice causes adverse effects on the surrounding environment and human health. To reduce the usage of herbicides, the distribution of weeds is sensed automatically by using various approaches. This aids in the optimization of the application dosage of the herbicides in the crop fields. This section explains about various conventional research works and methodologies used for the identification of weed from the plant leaf images.

Chaki et al. [2] proposed a novel method for characterizing and recognizing the plant leaves, with the combination of texture and shape features. The Gabor filter and GLCM were used for modeling the texture of the leaf, and a set of curvelet transform coefficients was used to capture the shape of the leaf along with the invariant moments. Improved recognition of the leaves with varying texture, size, shape and different orientations was achieved. Haug et al. [3] introduced a machine vision approach for plant classification without segmentation and discrimination of the crop/weed. A Random Forest classifier was used for the estimation of crop/weed certainty at sparse pixel positions, instead of segmenting the image. The proposed approach was evaluated using a set of images captured in the organic carrot farm. The average classification accuracy of the proposed approach was 
found to be high through the cross-validation of the images. Ahmed et al. [4] presented a simple and effective method for texture-based weed classification using local pattern operators. The performance of the proposed approach in the classification of weed images was found to be superior when compared to the existing method.

Kazmi et al. [5] addressed the problem of detecting thistle in sugar beet fields. Classification of species was performed using Mahalanobis Distance and Linear Discriminant Analysis. Highest detection accuracy was achieved through the effective selection of features using stepwise linear regression. Finally, the detection results were validated by annotating the images using the trained classifiers. Herrera et al. [6] proposed a method for discriminating between weeds based on the combined strategy. The main objective was to achieve appropriate discrimination between the two weed groups under varying conditions of lighting and soil background texture. Bo et al. [7] introduced a novel method of weed recognition based on the invariant moments. The features of the soybean leaf were obtained, and the soybean leaf was recognized using the nearest neighbor classifier. Effective recognition of weed was achieved and rapid, reliable and accurate identification of the weed location were enabled. Tahir et al. [8] proposed a novel method for processing the leaf images from the inconsistent illumination. The Radial Basis Function Network (RBFN) classifier was validated for the effective recognition of disease according to the type of deficiencies. The classification accuracy of the deficient leaf was improved.

Swain et al. [9] developed a smart weed identification technique based on the active shape modeling concept for the morphological identification of the crop and weed. The leaf model was aligned and deformed using automated active shape matching system. The nightshade plants were identified correctly within a short period. Ahmed et al. [10] investigated the usage of the machine-learning algorithm for the weed and crop classification. Testing of the features was performed to find the optimal combination of the significant features that ensures the highest classification rate. Rumpf et al. [11] focused on the automatic detection of various species with imaging sensors. A sequential classification approach including different SVM models was chosen for the classification of the weed and crop species. Early identification of the most harmful species was enabled, and optimal classification accuracy was improved. Torres-Sospedra and Nebot [12] introduced a weed detection procedure including two different stages. With the application of new noisy ensembles to the images, the location of the weeds was determined using the proposed procedure.

Siddiqi et al. [13] validated the accuracy of the wavelet transform method along with SVM and stepwise linear discriminant analysis for the classification of crops and weeds. The stepwise linear discriminant analysis was used for the extraction of the most significant features. Finally, the features were applied for the SVM classification. The classification results were compared with the existing techniques. The overall classification accuracy was improved. Liu et al. [14] developed a new algorithm for detecting the green plant using the hybrid spectral indices. The computation speed of the inter-row weed detection algorithm was higher than the Hough transformation method. Castillejo-González et al. [15] compared and tested the pixel-based and object-based techniques with different classification algorithms, for mapping the weed patches in the wheat fields using multi-spectral satellite image. The pixel-based classifications were applied to the wheat fields. Accurate discrimination of the weed patches was achieved.

Atkinson et al. [16] determined the utility of SVMs for detecting and mapping the presence of bug weed such as Solanum mauritianum within the Pinus patula plantations. Higher classification accuracy was achieved and the optimal subset of wavebands for the detection of bug weed was identified using SVM with recursive feature elimination approach. Garcia-Ruiz et al. [17] examined the feasibility of high-resolution imaging techniques for discriminating the sugar beet and thistle and proposed a multispectral camera filter selection protocol. From the simulation results, the improved correct classification rate of the thistles and sugar beets was recognized. Montalvo et al. [18] designed an automatic expert identification system based on image segmentation procedures, for the correct identification of plants. The identification performance of the system was improved irrespective of the loss of greenness of the plants. De Rainville [19] presented a method for classifying weeds and crops using the morphological analysis. The higher classification rate of the corn and soybean plants and weed was achieved.

Tannouche et al. [20] introduced an advanced technique to enable high herbicide saving and improved crop yield. The usage amount of herbicides was reduced, due to the localized spraying of the infected areas and efficient recognition of weeds. Tobal and Mokhtar [21] introduced an evolutionary Artificial NN (ANN) to minimize the time of classification training and error through the optimization of the neuron parameters. The classification accuracy was improved while avoiding the trial-and-error process of estimating the network inputs according to 
the histogram data. Mursalin and Mesbah-Ul-Awal [22] proposed an automated weed control system for the effective differentiation of the weeds and crops from the digital image. The classification of the weeds and crops according to the twelve extracted features. The computation cost was reduced and accuracy rate was improved. Yang et al. [23] developed a herbicide-spraying system in a corn field. The greenness level was computed based on the pixel-by-pixel based comparison of the red, green and blue intensity values of the image. The greenness percent of each image was obtained to generate weed coverage and patchiness maps. The maps were applied to the Fuzzy logic controller to determine application rate of herbicide in the corn field.

\section{Proposed ATP Extraction Method}

This section explains about the proposed ATP extraction method for crop and weed discrimination using curvelet transformation. AMF filters the impulse noise and applies the smoothening effect to the image for the clear analysis of textures. Green pixel extraction is performed, and green pixel count is calculated for vegetation extraction. K-means clustering is applied to the filtered image, to cluster the soil and plants. Then, the green pixel count is verified with the pixel count of the clusters. If the green pixel count is nearest to the pixel count of the clusters, then it is considered as the plant image. Wrapping based curvelet transform is applied to represent the smooth edges and other singularities along the curve more efficiently than the wavelet transform. The problem of the edge discontinuity is better approximated by the curvelets than the wavelets. Curvelets can solve the curved singularity representation and limited orientation of the existing wavelet transform. The speed of the curvelet transform is high, due to the lower amount of redundancy in the curvelet transform. Wrapping based curvelet transform utilizes a series of translations to ensure faster computation capability. After applying the curvelet transformation, the angular based features are extracted from the transformed image.

Feature extraction is performed to extract the angular texture pattern of the plant image. In the texture pattern analysis, an MWP system is used to predict and analyze the difference in texture in the image. The Tamura features are extracted from the image, Gabor filter with 4 orientations is applied to the image and GLCM features are extracted from the Gabor filtered image. PSO-based DEFS is applied to select the optimal features. Then, the selected features are learned and passed through an RVM-based classifier to find out the weed. Canny-based Edge detection and contouring is performed based on the classification results, to identify the weed. Clear analysis and segmentation of the plant image in the complex background are achieved due to the angular texture pattern extraction at each windowing patch. The overall flow diagram of the proposed ATP extraction method is shown in Figure 1. The proposed method involves the following process

- Pre-processing

- Plant image identification

- Transformation

- Feature Extraction

- Feature Selection

- Classification

- Weed Identification

- Weed Patchiness Level Detection 


\subsection{Adaptive Median Filtering}

Pre-processing of the plant image is performed by using the AMF, to remove the noise and correct the distortions in the image. The standard median filter cannot distinguish the fine detail from noise. The AMF is designed to overcome the drawbacks of the standard median filter. In the AMF, the size of the window that surrounds each pixel in the image is variable depending on the median value of the pixels in the current $3 * 3$ window. If the median value is an impulse, the size of the window is expanded. Otherwise, further image processing is done within the current window specifications. The center pixel of the window is estimated to verify whether the center pixel is an impulse or not. The new value of the center pixel in the filtered image is the median value of the pixel in the window if the center pixel is an impulse. The center pixel value is retained in the filtered image if the center pixel is not an impulse. Thus, the grayscale value of the pixels in the input image and filtered image becomes same, unless the pixel is considered as an impulse. Hence, the AMF removes the impulse noise from the image and reduces distortion in the image.

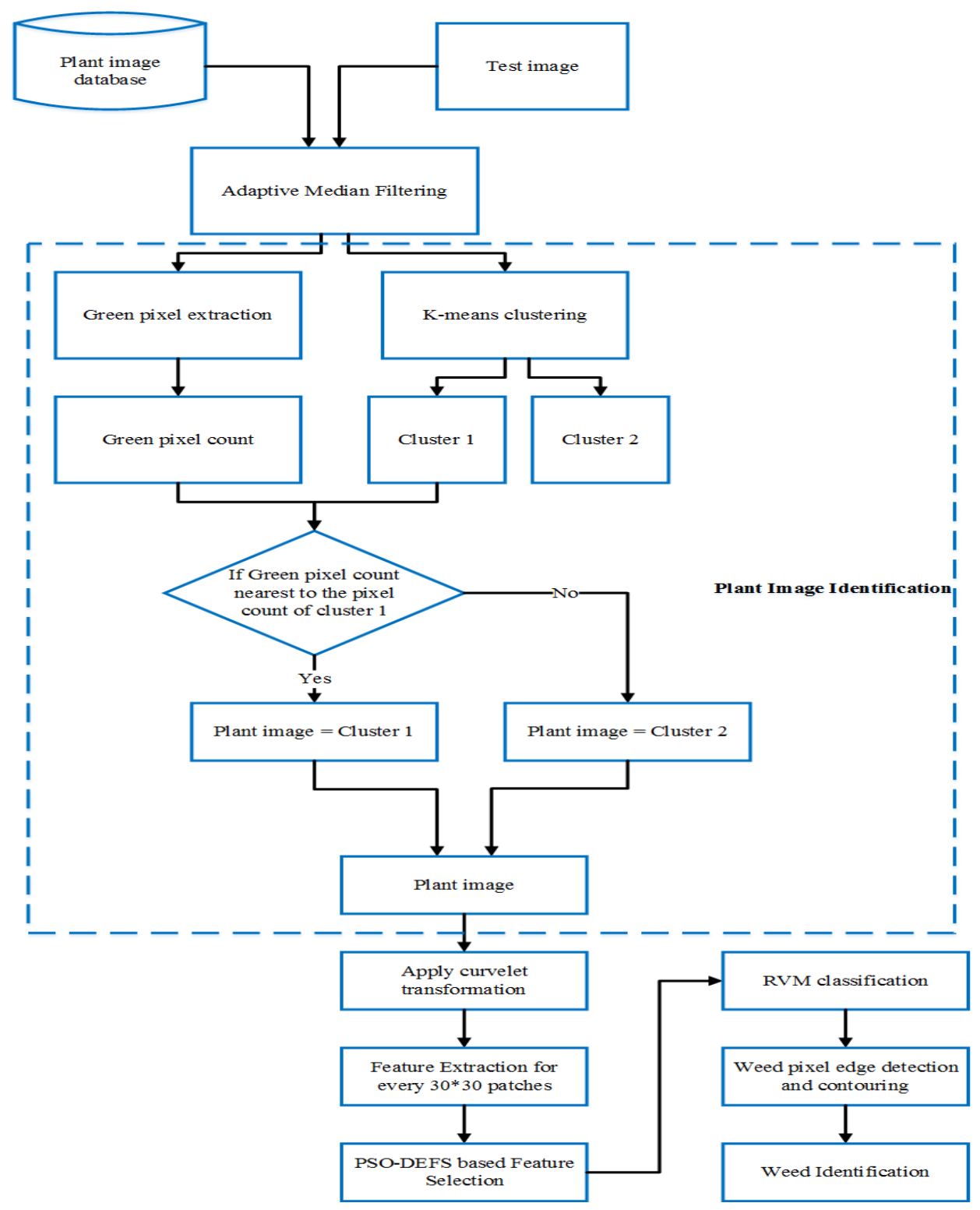

Figure 1: Overall flow diagram of the proposed ATP extraction method 
The AMF approach includes two levels. The first level determines whether the output of the median filter $\mathrm{Z}_{\mathrm{MED}}$ is an impulse output or not. If the first level fails to find the impulse output, then the second level is executed. The first level is repeated until a median value is found out, or the maximum window size is obtained. The gray level value is obtained from the first level. For each output produced by the algorithm, the window is subsequently moved to the following location in the image. Then, the filtering algorithm is reinitialized again and applied to the pixels in the new location. The median values are updated iteratively using the new pixels, thus reducing the computational overhead. The adaptive median filtering algorithm is described as given below

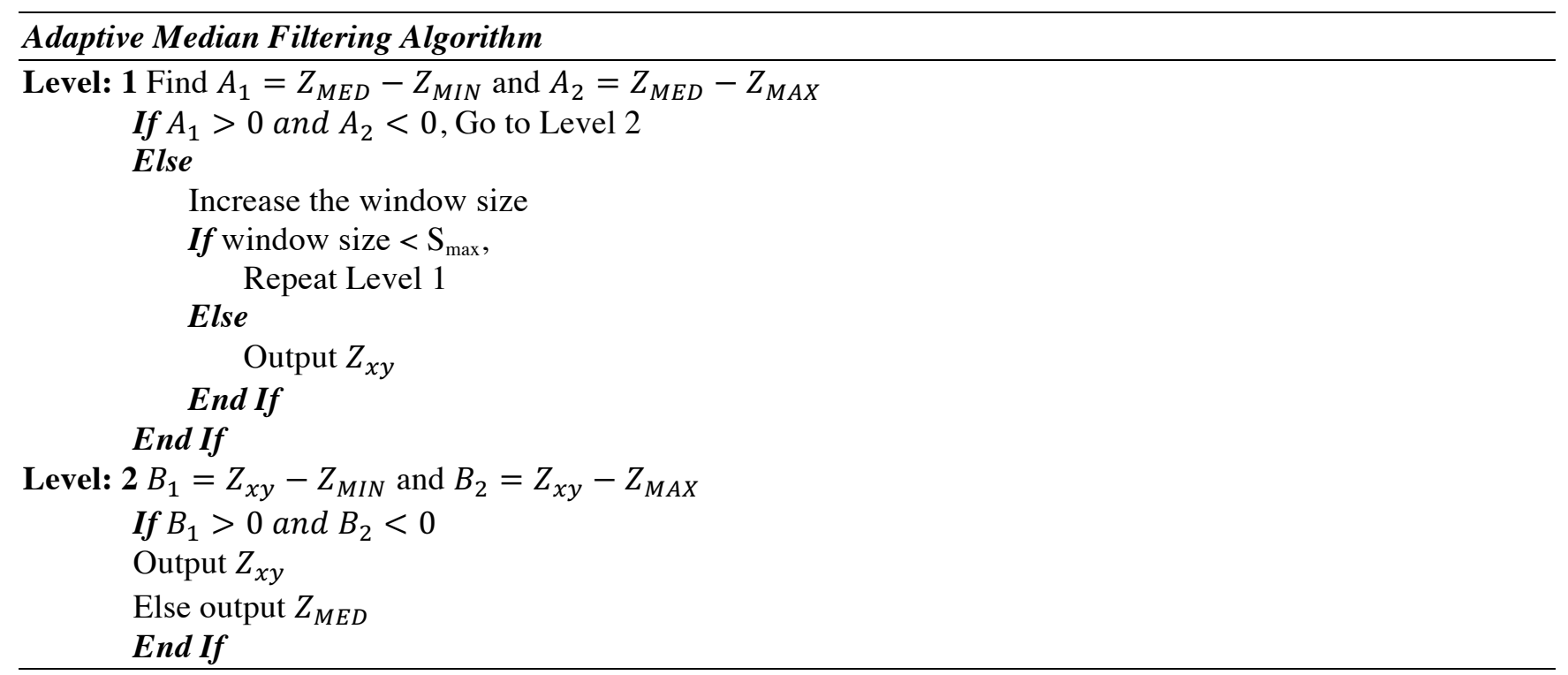

Where ' $\mathrm{S}_{\max }$ ' denotes the maximum allowed size of $\mathrm{S}_{\mathrm{xy}}$ and ' $\mathrm{S}_{\mathrm{xy}}$ ' denotes the size of the neighborhood. $Z_{M E D}$ is the median of gray levels in $\mathrm{S}_{\mathrm{xy}}, Z_{M I N}$ is the minimum gray level value, $Z_{M A X}$ is the maximum gray level value, and $Z_{x y}$ is the gray level at coordinates (x,y). Figure.2 (a) shows the original image of dataset 1 and Figure.2 (b) shows the original image of dataset 2. Figure.3 (a) and Figure.3 (b) show the pre-processed images of dataset 1 and dataset 2 .

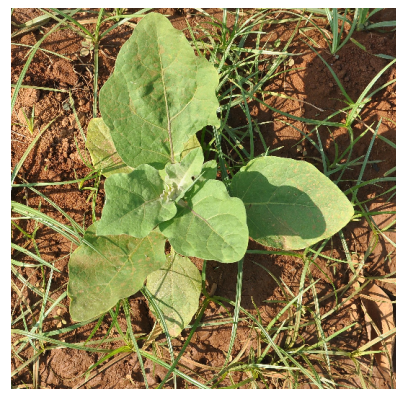

(a)

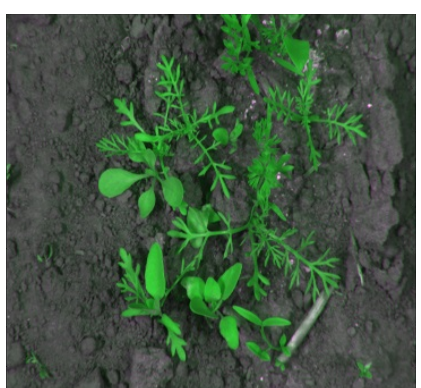

(b)

Figure 2: (a) and (b) Original images of dataset 1 and dataset 2 


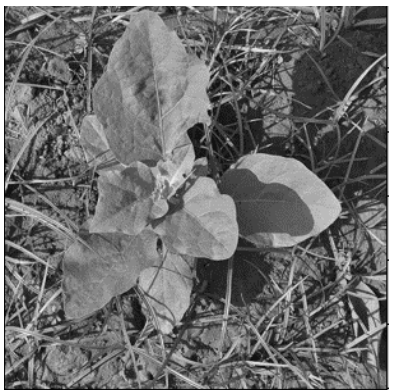

(a)

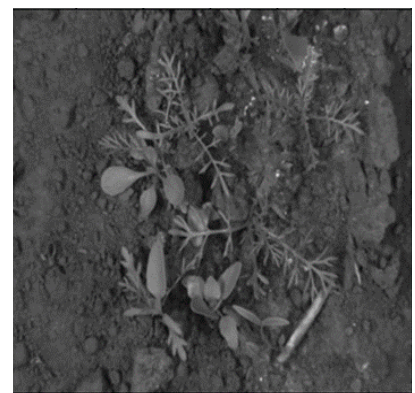

(b)

Figure 3: (a) and (b) Pre-processed images of dataset 1 and dataset 2

\subsection{Plant Image Identification}

The plant image is identified using the green pixel extraction and k-means clustering process.

\subsubsection{Green Pixel Extraction}

After filtering the input image, extraction of the green pixel in the plant image is performed for the clear recognition of the plant. Green pixel extraction is performed, and green pixel count is calculated for vegetation extraction. The green pixel count [24] is computed using the formula

$$
P_{C}=2 G-R * G-B
$$

$P_{C}$ denotes the green pixel count. $\mathrm{R}, \mathrm{G}$, and $\mathrm{B}$ are the three components of pixel color in RGB color space. The pixel values of the red channel and blue channel are less than the pixel values of the green channel. Figure.4 (a) and Figure.4 (b) shows the green pixel extraction process of dataset 1 and dataset 2.

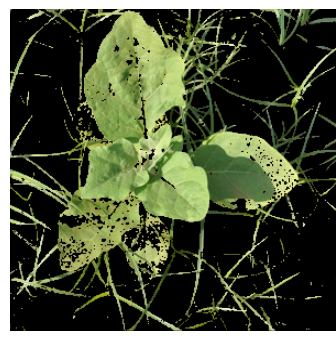

(a)

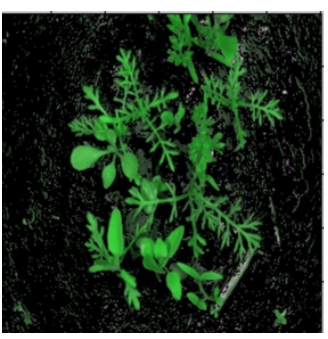

(b)

Figure 4: (a) and (b) Green Pixel Extraction of dataset 1 and dataset 2

\subsubsection{K-means Clustering}

Since the RGB color space does not consider the human perception into account, the CIELAB color space is used in our proposed work to approximate the human vision. The CIELAB color space is used to make accurate color balance corrections or adjust the contrast using the lightness component. The conversion from RGB values to the LAB values involves the transformation of the RGB values to an absolute color space represented as sRGB. Once the colors are converted to sRGB, they are first converted to linear sRGB values and then to the CIE XYZ values. Finally, conversion of colors to the CIE LAB color space is performed using a D65 standard illuminant. Then, the grouping of the pixels into the clusters of dominant colors is performed using a standard K-means clustering algorithm. The $K$-means clustering is applied to the filtered image, for separating the soil and plants. It is one of the most frequently used techniques for the automatic partitioning of a data set into ' $\mathrm{k}$ ' groups. According 
to the algorithm, the ' $\mathrm{k}$ ' objects are selected as initial cluster centers and the distance between each object and each cluster center is calculated. The object is assigned to the nearest cluster, and the average of all clusters is updated. $K$-means clustering performs partitioning of data into the $k$ mutually exclusive clusters, and then returns the index of the cluster assigned to each observation. In our proposed work, K-means clustering is used for clustering the filtered image.

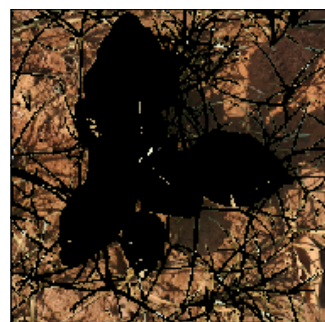

(a)

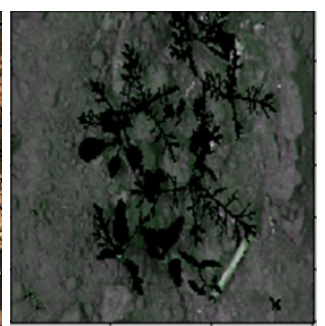

(b)

Figure 5: (a) and (b) Objects in cluster 1 of dataset 1 and dataset 2

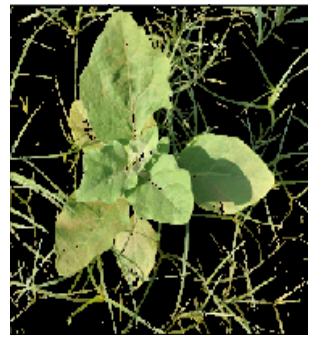

(a)

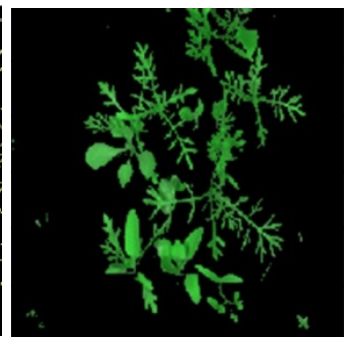

(b)

Figure 6: (a) and (b) Objects in cluster 2 of dataset 1 and dataset 2

Figure.5 (a) and Figure.5 (b) show the objects in the Clusters 1 of the dataset 1 and dataset 2. Figure.6 (a) and Figure.6 (b) shows the objects in the cluster 2 of the dataset 1 and dataset 2 . Then, the green pixel count is verified with the pixel count of the clusters. If the green pixel count is nearest to the pixel count of the clusters, then it is considered as the plant image. The plant images of the dataset 1 and dataset 2 are shown in Figure.7 (a) and Figure.7 (b).

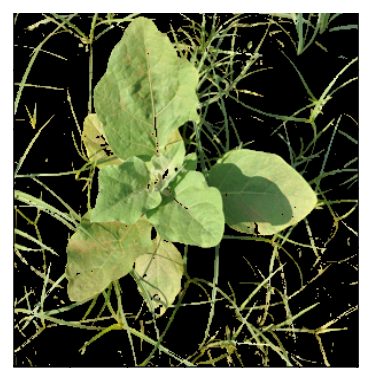

(a)

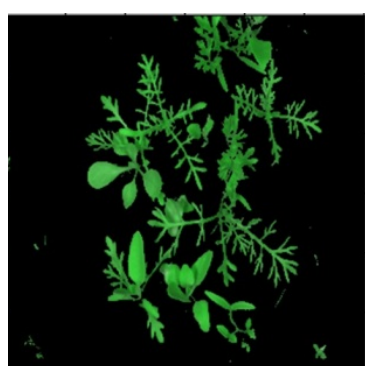

(b)

Figure 7: (a) and (b) Plant images of dataset 1 and dataset 2

\subsection{Curvelet Transformation}

Curvelet transformation is applied for representing the images at different scales and angles. In our work, wrapping-based curvelet transform is applied to the plant image, to obtain the forward and backward curvelet 
transformation. Wrapping based curvelet transform is a multi-scale pyramid that consists of different orientations and positions at a low-frequency level. The wrapping algorithm requires low computation time by using a series of translations and a wraparound technique. The merits of the Fast Fourier Transform (FFT) are used for the multiresolution discrete curvelet transform in the spectral domain. The image and curvelet at a given scale and orientation are transformed into the Fourier domain, during FFT. A set of curvelet coefficients is obtained by applying inverse FFT to the spectral product, at the completion of the computation process. The curvelet coefficients in the ascending order of the scales and orientations are included in the set. The frequency response of the curvelet is in the form of the trapezoidal wedge. For the inverse Fourier transform, wrapping of the frequency response of the curvelet into a rectangular support is performed. Wrapping of this trapezoidal wedge is done by periodically tiling the spectrum inside the wedge and collecting the rectangular coefficient area in the origin. With the periodic tiling, the rectangular region collects the corresponding fragmented portions of the wedge from the surrounding parallelograms.

The wrapping-based curvelet transform involves the following steps:

1. Obtain the Fourier Samples by applying the FFT $\hat{F}\left(n_{1}, n_{2}\right)$ Where $-n / 2 \leq n_{1}, n_{2}<n / 2$

2. Generate the product for each scale ' $m$ ' and angle ' $\theta$ '

$\hat{C}_{m, \theta}\left[n_{1}, n_{2}\right] \hat{F}\left[n_{1}, n_{2}\right]$, where 'C' denotes the Cartesian window

3. Wrap this product about the origin and obtain $\hat{F}_{m, \theta}\left[n_{1}, n_{2}\right]=W\left(\hat{C}_{m, \theta} \hat{F}\right)\left[n_{1}, n_{2}\right]$, where the range of $n_{1}$ and $n_{2}$ is $0 \leq n_{1}<K_{1, m}$ and $0 \leq n_{2}<K_{2, m}$.' $\theta$ ' is the range of $(-\pi / 4, \pi / 4)$.

4. Apply inverse FFT to the $\hat{F}_{m, \theta}$, thus collect the discrete coefficients $D(m, \theta, p)$, where 'p' is the spatial location parameter.

The number of curvelet decomposition levels for the curvelet transform is determined using the following formula

$$
N=\log _{2}(\operatorname{Size}(M, 1))-2
$$

Where ' $N$ ' is the number of levels and ' $M$ ' is the number of rows. The size of the plant image is $300 * 300$. By applying the size in the eqn (2), the number of levels for the curvelet transformation is 6 . Hence, six levels of curvelet decomposition are applied to the input image. Overall three mean values are obtained from the curvelet transformation. Figure.8 (a) and Figure.8 (b) show the curvelet transformed image of the dataset 1 and dataset 2. Figure. 9 shows the Graphical User Interface (GUI) image for curvelet transformation process.

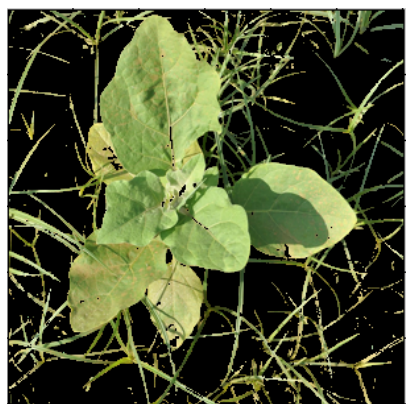

(a)

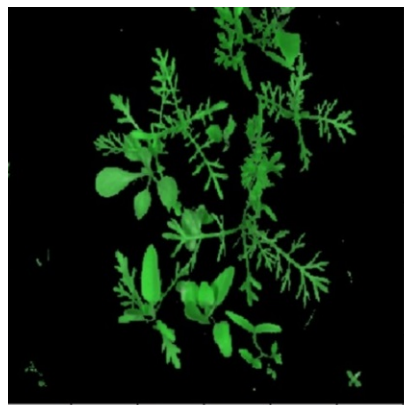

(b)

Figure 8: (a) and (b) Curvelet transformed image of dataset 1 and dataset 2 


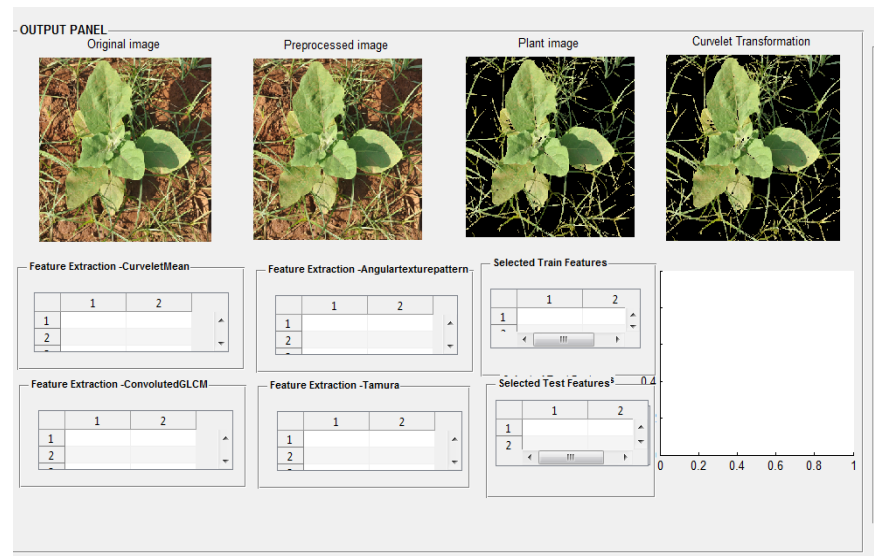

Figure 9: GUI Image for Curvelet transformation process

\subsection{Feature extraction}

Feature extraction is the process of defining a set of features, for the efficient representation of the information for analysis and classification. Angular texture pattern is extracted from the curvelet transformed image. The Tamura features are extracted from the image, Gabor filter with four orientations is applied to the image and GLCM features are extracted from the Gabor filtered image. Figure.10 and Figure.11 show the separated patches of the image of the dataset 1 and dataset 2 . Figure.12 shows the main stages of the feature extraction process.

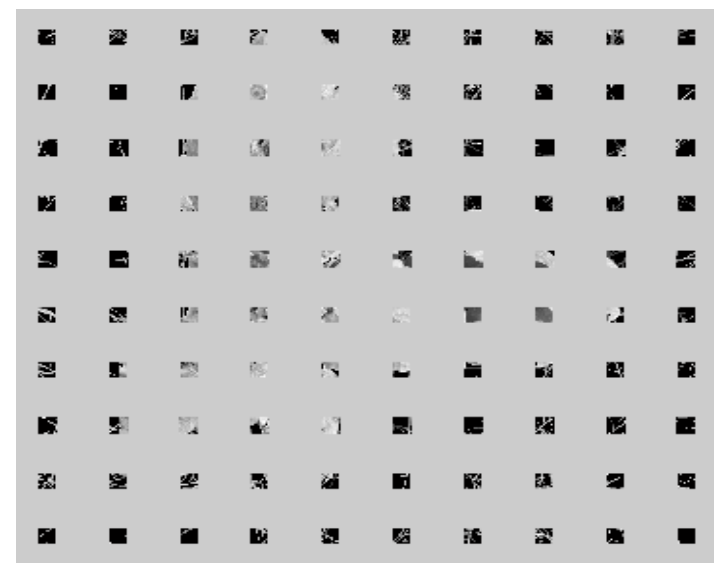

Figure 10: Patch Separation for dataset 1

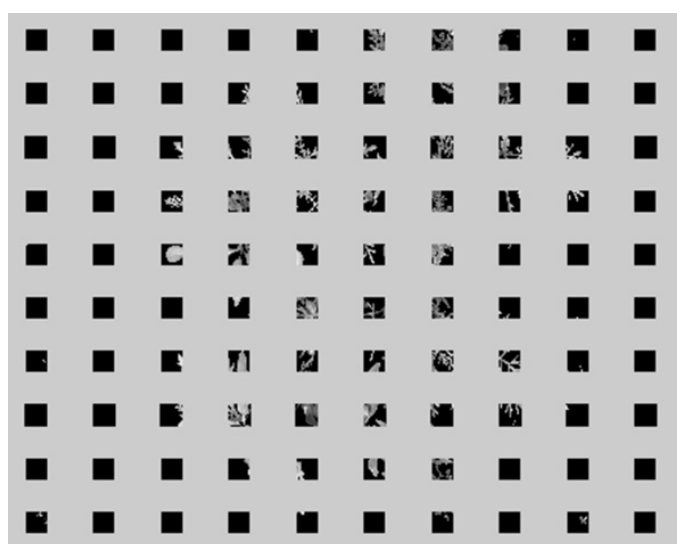

Figure 11: Patch Separation for dataset 2 


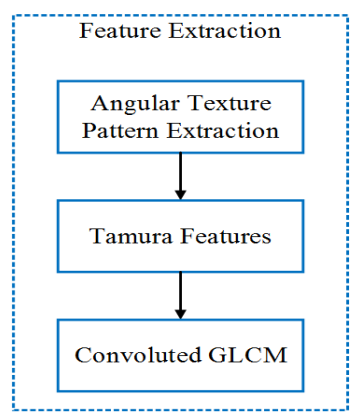

\subsubsection{ATP extraction}

Figure 12: Main stages of Feature extraction process

After applying the curvelet transformation, the angular based features are extracted from the transformed image. The ATP extraction algorithm is described below:

\section{ATP Extraction Algorithm}

\section{Input: Image}

Output: Texture pattern image

Divide Image into $3 * 3$ window

Calculate Average of diagonal pixels

For $\mathrm{i}=2$ to $\mathrm{m}-1$

For $\mathrm{j}=2$ to $\mathrm{n}-1$

Temp = im (i-1: i+1, j-1: j+1, cc);

$\mathrm{t}_{\mathrm{s}}=\operatorname{mean}([\operatorname{temp}(1)$ temp $(3) \operatorname{temp}(7) \operatorname{temp}(9)$ temp $(5)])$;

$/ /$ calculate average of center and diagonal pixels

Temp 1(1) $=\mathrm{im}(\mathrm{i}-1, \mathrm{j}-1)>\mathrm{t}_{\mathrm{s}}$;

Temp 1(2) $=\mathrm{im}(\mathrm{i}-1, \mathrm{j})>\mathrm{t}_{\mathrm{s}}$;

Temp 1(3) $=\mathrm{im}(\mathrm{i}-1, \mathrm{j}+1)>\mathrm{t}_{\mathrm{s}}$;

Temp 1(4) $=\mathrm{im}(\mathrm{i}, \mathrm{j}+1)>\mathrm{t}_{\mathrm{s}}$;

Temp 1(5) = im $(i+1, j+1)>t_{s}$;

Temp 1(6) $=\mathrm{im}(\mathrm{i}+1, \mathrm{j})>\mathrm{t}_{\mathrm{s}}$;

Temp 1(7) $=\mathrm{im}(\mathrm{i}+1, \mathrm{j}-1)>\mathrm{t}_{\mathrm{s}}$;

Temp 1(8) $=\mathrm{im}(\mathrm{i}, \mathrm{j}-1)>\mathrm{t}_{\mathrm{s}}$;

Temp 1(9) $=\mathrm{im}(\mathrm{i}, \mathrm{j})>\mathrm{t}_{\mathrm{s}}$;

Count $=0$;

Count $1=0$

For $\mathrm{i}=1: 9$

For temp $(\mathrm{i},:)==1$;

Count=count +1 ;

Else

Count $1=$ count $1+1$;

End For

End For

\section{End For}

\section{End For}

Pattern $=$ [count 1 count $]$;

Replace all pixels by the new pattern

Get the texture pattern image 
The main limitations of the Local Binary Patterns (LBP) are the sensitivity of the long histograms to the image rotation, the small spatial area of support, loss of local textural information and sensitivity to noise. Hence, the extraction of ATP is performed to overcome the limitations of the LBP. In the texture pattern analysis, a novel system of MWP system is presented to predict and analyze the difference in the texture of the image. In the window projection method, the texture pattern is extracted using the enhanced model of the angular texture pattern extraction method.

In the angular texture pattern extraction method, the images are divided into patches, such that each patch contains a $3 * 3$ window. Then, the diagonal pattern is applied. The window is divided into $3 * 3$ cells. The average of diagonal pixels is calculated and replaced with all diagonal pixels. When the average pixel value is greater than or equal to the neighbor pixel value, then the value of ' 1 ' is written. Otherwise, the value of ' 0 ' is written. Thus, an 8-digit binary number is obtained. Usually, this binary value is converted to decimal for convenience. By counting the number of zeroes and ones, the pattern count of ones placed in left and count of zeroes placed in the right are generated. The histogram related to the frequency of occurrence of each number is computed. The histogram is normalized, and all the cells of the normalized histograms are concatenated. Finally, the angular texture pattern feature vector is obtained for the window. In the feature extraction step, level 2 and level 5 subband coefficients are selected for the extraction of features in six-level curvelet decomposition of the images. The level 2 coefficients include the finer details and level 5 coefficients include the coarse details of the image [25].

Where, ' $t_{s}$ ' represents the average value of the diagonal and center pixel. If the neighbor value of the center pixel is greater than the average value, it is considered as ' 1 ' otherwise ' 0 '. In our proposed work, the pattern is extracted from the input image based on the diagonal pixel values. Figure. 13 shows the $3 * 3$ matrix for the texture pattern image and Figure.14 illustrates the calculation of the average values of the diagonal pixels in the $3 * 3$ matrix.

\begin{tabular}{|l|l|l|}
\hline 3 & 4 & 5 \\
\hline 6 & 7 & 8 \\
\hline 9 & 10 & 11 \\
\hline
\end{tabular}

Figure 13: $3 * 3$ Matrix for texture pattern image

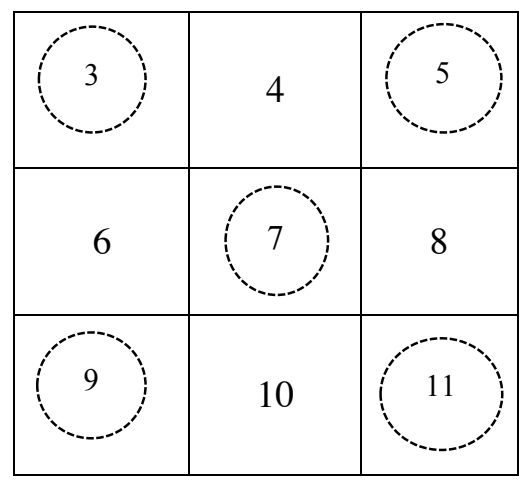

Figure 14: Average calculation of the diagonal pixel values in the $3 * 3$ matrix 
The average of the values $3,5,7,9,11$ is $3+5+9+11+7=35 / 5=7$.

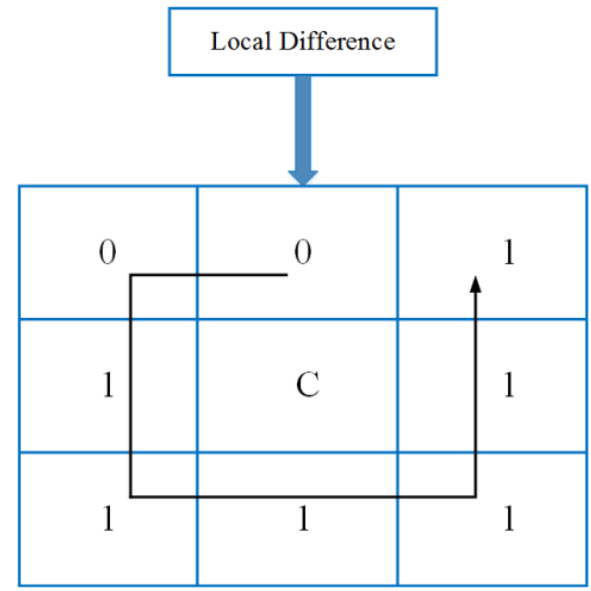

Figure 15: Pattern generation using the proposed ATP extraction process

The original LBP operator labels the pixels in the image by thresholding the neighborhood value of each pixel with the center pixel value and considering the result as a binary number. Figure. 15 explain about the pattern generation by the proposed angular pattern extraction process. The binary value 0011111 for this pattern contains two zeroes and five ones. Hence, the pattern 35 is generated. Likewise, the patterns are generated.

\subsubsection{Tamura Features}

After extraction of the angular texture pattern, the Tamura features are extracted from the image. The Tamura features characterize the low-level statistical properties of the images. The Tamura features are discussed as follows

a) Coarseness $\left(F_{c o a}\right)$

Coarseness is a measure of the size of the texture elements. It provides us with the information about the size of the coarse and fine textures elements. Fine textures have smaller coarse value than the coarse textures.

$$
F_{c o a}=\frac{2^{q}}{M^{2}} \sum_{i}^{M} \sum_{j}^{M} P(i, j)
$$

Where $M \times M$ denotes the size of the image, $\mathrm{P}(\mathrm{i}, \mathrm{j})$ is the sum of each pixel, and ' $\mathrm{q}$ ' is the difference of the moving averages.

b) Contrast $\left(F_{\text {con }}\right)$

Contrast defines the difference in the intensity value among the neighboring pixels. The image contrast is influenced by the dynamic range of gray levels in the image, polarization of the distribution of the black and white, sharpness of edges and period of repeating patterns. It could also stand for picture quality in the narrow sense.

$$
F_{\text {Con }}=\frac{\sigma}{\sqrt[4]{\gamma_{4}}}
$$

Where

$$
\gamma_{4}=\frac{\mu_{4}}{\sigma^{4}}
$$

Here ' $\mu_{4}$ ' denotes the fourth moment about the mean ' $\mu$ ', and $\sigma^{2}$ denotes the variance. 
c) Directionality $\left(F_{\text {dir }}\right)$

Directionality measures the total degree of directionality, and also differentiates between the different orientations or patterns.

$$
F_{\text {dir }}=1-N_{f} \cdot N_{p} \cdot \sum_{P}^{N_{p}} \sum_{Q \in R_{P}}\left(Q-Q_{p}\right)^{2} \cdot H_{D}(Q)
$$

Where ' $N_{p}$ ' is the number of peaks in the histogram, 'Q' is the quantized direction code, ' $Q_{p}$ ' is the $\mathrm{p}^{\text {th }}$ peak position of the histogram, ' $R_{P}$ ' is the range of the $\mathrm{p}^{\text {th }}$ peak between the valleys and ' $N_{f}$ ' is the normalizing factor.

d) Line - Likeness $\left(F_{\text {lin }}\right)$

Line-Likeness is defined as the average coincidence of the edge directions that co-occur at pixels separated by a distance ' $\mathrm{D}$ ' along the direction.

$$
F_{\text {lin }}=\left\{\begin{array}{c}
\frac{\sum_{\mathrm{a}=0}^{\mathrm{n}-1} \sum_{\mathrm{b}=0}^{\mathrm{n}-1} \mathrm{P}_{\mathrm{D}}(\mathrm{a}, \mathrm{b}) \operatorname{Cos}\left[(\mathrm{a}-\mathrm{b}) \frac{2 \pi}{\mathrm{n}}\right]}{\sum_{\mathrm{a}=0}^{\mathrm{n}-1} \sum_{\mathrm{b}=0}^{\mathrm{n}-1} \mathrm{P}_{\mathrm{D}}(\mathrm{a}, \mathrm{b})} \text { if } \sum_{\mathrm{a}=0}^{\mathrm{n}-1} \sum_{\mathrm{b}=0}^{\mathrm{n}-1} \mathrm{P}_{\mathrm{D}}(\mathrm{a}, \mathrm{b})>\mathrm{t} \\
0 \text { Otherwise }
\end{array}\right.
$$

e) Regularity $\left(F_{\text {reg }}\right)$

The degree of the irregularity is calculated using the equation

$$
\text { Freg }=1-\eta(\sigma c o a+\sigma c o n+\sigma d i r+\sigma r e g)
$$

' is the normalizing factor and ' $\sigma$ ' means the standard deviation of the feature in each subimage of the texture.

f) Roughness $\left(F_{\text {rou }}\right)$

Roughness represents the concrete variations in the texture of the image.

$$
F_{\text {rou }}=F_{\text {coa }}+F_{\text {con }}
$$

In our proposed work, energy, entropy and auto correlation values are calculated additionally.

g) $\operatorname{Energy}\left(F_{E N}\right)$

Energy is the measure of the uniformity of the gray distribution level of the image.

$$
F_{\mathrm{EN}}=\sum_{\mathrm{i}} \sum_{\mathrm{j}} \mathrm{P}^{2}(\mathrm{i}, \mathrm{j})
$$

Where ' $P$ ' denotes the probability values for the gray scale pixels ' $i$ ' and ' $\mathrm{j}$ '.

h) $\operatorname{Entropy}\left(F_{H}\right)$

Entropy is the measure of the amount of the texture information of an image. 


$$
F_{H}=\sum_{\mathrm{i}} \sum_{\mathrm{j}} \mathrm{P}(\mathrm{i}, \mathrm{j}) \log \mathrm{P}(\mathrm{i}, \mathrm{j})
$$

i)

Auto-Correlation $\left(F_{A C}\right)$

The auto-correlation is used to measure the degree of similarity of the elements in the image. It decreases quickly and offers a lot of variations, for the images with the low degree of coarseness.

$$
F_{A C}=1-n_{f}(\text { kurtosis }(\text { con })+\operatorname{kurtosis}(\text { dir })+\operatorname{kurtosis}(\operatorname{lin})+\operatorname{kurtosis}(\text { rou }))+F_{\text {rou }}
$$

The auto-correlation is computed based on the contrast, directionality, line-linkedness and roughness values.

\subsubsection{Convoluted GLCM}

Gabor filter is the most popular tool for feature extraction. It includes the convolution of an image using several multi-orientation filters. In our proposed work, the image is convoluted along four different orientations. The Gabor filter is used to obtain the clear texture of the image. Transformed space is created for each convolution, and the feature extraction is done in each transformed space. The features vector includes the energy measure of each convoluted image. The Gabor filter is the product of the Gaussian Kernel and the complex sinusoid. The Gaussian curve is represented as

$$
G(x, y, \sigma)=\frac{1}{2 \pi \sigma^{2}} \cdot \exp \left(-\frac{x^{2}+y^{2}}{2 \sigma^{2}}\right)
$$

The complex sinusoid is defined as follows

$$
s(x, y, u, \theta, \varphi)=\exp \{j 2 \pi(x \cdot u \cos \theta+y \cdot u \sin \theta)+\varphi\}
$$

Where ' $u$ ' denotes the spatial frequency, ' $\theta$ ' denotes the orientation and ' $\varphi$ ' represents the phase shift. The complex Gabor function ' $\mathrm{H}$ ' is given as

$$
H(x, y, u, \theta, \varphi)=G(x, y, \sigma) \cdot s(x, y, u, \theta, \varphi)
$$

The input image $\mathrm{I}(\mathrm{x}, \mathrm{y})$ is convolved with the Gabor filter ' $\mathrm{H}$ ' to produce a set of complex signals ' $\mathrm{C}$ '.

$$
C(x, y)=I(x, y) \otimes H(x, y, u, \theta, \varphi)
$$

The real and imaginary parts of the image are separated as given below

$$
\begin{aligned}
& R(x, y)=\operatorname{Re}\{J(x, y)\} \\
& I(x, y)=\operatorname{Im}\{J(x, y)\}
\end{aligned}
$$

GLCM features are extracted from the Gabor filtered image. GLCM is defined as a square matrix that defines the spatial distribution of the gray levels in the image. The GLCM is used to compute a set of scalar quantities that characterize the different aspects of the texture in the image. It defines about the linear relationship between the reference pixel ' $i$ ' and neighboring pixel ' $j$ ' located within the area of interest. Each element $(a, b)$ is the number of co-occurrences of the pixels. The pixels are located at a distance ' $D$ ' with respect to each other. The GLCM is computed by using the equation 


$$
C_{a, b, \varphi}=\sum_{\mathrm{i}=1}^{\mathrm{M}} \sum_{\mathrm{j}=1}^{\mathrm{M}}\left\{\begin{array}{lr}
1, & \text { if } \mathrm{I}(\mathrm{i}, \mathrm{j})=\mathrm{a} \text { and } \mathrm{I}\left(\mathrm{i}+\varphi_{i}, \mathrm{j}+\varphi_{j}\right)=\mathrm{b} \\
0, & \text { Otherwise }
\end{array}\right.
$$

Where ' $\varphi_{i}$ ' and ' $\varphi_{j}$ ' denote the relative orientations of the pixels ' $\mathrm{i}$ ' and ' $\mathrm{j}$ '. ' a' represents the gray level of the pixels $(i, j)$ and 'b' represents the gray level of the pixels $\left(i+\varphi_{i}, j+\varphi_{j}\right)$. During the analysis of statistical texture, computing of the texture features is performed from the statistical distribution of the observed combinations of intensities at specified positions in the image. GLCM method is a method of extracting the second order statistical texture features. The number of rows and columns in the GLCM matrix is equal to the number of gray levels ' $\mathrm{G}$ ' in the image. The pixels are separated by the distances $\delta_{x}$ and $\delta_{y}$ within a given neighborhood. The matrix element $\mathrm{P}\left(\mathrm{i}, \mathrm{j} \mid \delta_{x}, \delta_{y}\right)$ is the relative frequency with which the pixels are separated. The gray levels of the pixels are ' $\mathrm{i}$ ' and ' $\mathrm{j}$ '. The matrix element $\mathrm{P}(\mathrm{i}, \mathrm{jld}, \theta)$ includes the second order statistical probability values for the difference between the gray levels at a particular displacement distance ' $d$ ' and at an angle ' $\theta$ '. In our proposed work, displacement distance $=1$ and direction $(\theta)=0,45,90,135$ degree. The GLCM features are described below

$$
\begin{gathered}
\mathrm{F}_{\mathrm{ENG}}=\sum_{\mathrm{i}=1}^{\mathrm{N}} \sum_{\mathrm{j}=1}^{\mathrm{N}}\left\{S(\mathrm{i}, \mathrm{j})^{2}\right\} \\
\mathrm{F}_{\mathrm{CON}}=\sum_{\mathrm{i}=1}^{\mathrm{N}} \sum_{\mathrm{j}=1}^{\mathrm{N}}\left[(\mathrm{i}-\mathrm{j})^{2} * \mathrm{~S}(\mathrm{i}, \mathrm{j})\right] \\
F_{C O R R}=\frac{1}{\sigma^{2}}\left\{\left(\sum_{\mathrm{i}}^{\mathrm{N}} \sum_{\mathrm{j}}^{\mathrm{N}} \mathrm{i} \cdot \mathrm{j} \cdot \mathrm{S}(\mathrm{i}, \mathrm{j})\right)-\mu^{2}\right\} \\
\mathrm{F}_{\mathrm{VAR}}=\sum_{\mathrm{i}=1}^{\mathrm{N}} \sum_{\mathrm{j}=1}^{\mathrm{N}}(\mathrm{i}-\mu)^{2} \mathrm{~S}(\mathrm{i}, \mathrm{j}) \\
F_{H O M}=\sum_{\mathrm{i}=1}^{\mathrm{N}} \sum_{\mathrm{j}=1}^{\mathrm{N}} \frac{\mathrm{S}(\mathrm{i}, \mathrm{j})}{1+(\mathrm{i}-\mathrm{j})^{2}} \\
F_{A V G}=\sum_{\mathrm{i}=2}^{2 \mathrm{~N}} i \cdot P_{x y}(\mathrm{i}) \\
\mathrm{f}_{S e}(\mathrm{i})=\mathrm{P}_{\mathrm{xy}}(\mathrm{i}) \log _{\mathrm{e}} \mathrm{P}_{\mathrm{xy}}(\mathrm{i}) \\
P_{x y}(\mathrm{i}+\mathrm{j})=\sum_{\mathrm{i}=1}^{2 \mathrm{~N}} \sum_{\mathrm{j}=1}^{2 \mathrm{~N}} \mathrm{~S}(\mathrm{i}, \mathrm{j}) \\
\mathrm{F}_{\mathrm{SE}}=-\mathrm{f}_{s v}(\mathrm{i})
\end{gathered}
$$




$$
\begin{aligned}
& \mathrm{f}_{\mathrm{sv}}(\mathrm{i})=\left(i-F_{E N T}\right)^{2} \cdot \mathrm{P}_{\mathrm{xy}}(\mathrm{i}) \\
& \mathrm{F}_{\mathrm{ENT}}=-\sum_{\mathrm{i}=1}^{\mathrm{N}} \sum_{\mathrm{j}=1}^{\mathrm{N}} \mathrm{S}(\mathrm{i}, \mathrm{j}) \cdot \log _{\mathrm{e}} \mathrm{S}(\mathrm{i}, \mathrm{j}) \\
& P_{x}(\mathrm{i})=\sum_{\mathrm{j}=1}^{\mathrm{N}} \mathrm{S}(\mathrm{i}, \mathrm{j}) \\
& P_{y}(\mathrm{j})=\sum_{\mathrm{i}=1}^{\mathrm{N}} \mathrm{S}(\mathrm{i}, \mathrm{j}) \\
& h_{x}(\mathrm{i})=\mathrm{P}_{\mathrm{x}}(\mathrm{i}) \cdot \log _{\mathrm{e}} \mathrm{P}_{\mathrm{x}}(\mathrm{i}) \\
& h_{y}(j)=\mathrm{P}_{y}(\mathrm{j}) \cdot \log _{\mathrm{e}} \mathrm{P}_{y}(\mathrm{j}) \\
& h_{x y 1}(\mathrm{i}, \mathrm{j})=\mathrm{S}(\mathrm{i}, \mathrm{j}) \cdot \log \left[\mathrm{P}_{\mathrm{x}}(\mathrm{i}) \cdot \mathrm{P}_{y}(\mathrm{j})\right] \\
& h_{x y 2}(\mathrm{i}, \mathrm{j})=\mathrm{P}_{\mathrm{x}}(\mathrm{i}) \cdot \mathrm{P}_{y}(\mathrm{j}) \cdot \log \left[\mathrm{P}_{\mathrm{x}}(\mathrm{i}) \cdot \mathrm{P}_{y}(\mathrm{j})\right] \\
& \mathrm{H}_{\mathrm{xy}}=\mathrm{F}_{\mathrm{ENT}} \\
& \mathrm{H}_{\mathrm{X}}=-\sum_{\mathrm{i}=1}^{\mathrm{N}} \mathrm{h}_{\mathrm{x}}(\mathrm{i}) \\
& \mathrm{H}_{\mathrm{Y}}=-\sum_{\mathrm{i}=1}^{\mathrm{N}} \mathrm{h}_{\mathrm{y}}(\mathrm{j}) \\
& \mathrm{H}_{\mathrm{XY} 1}=-\sum_{\mathrm{i}=1}^{\mathrm{N}} \sum_{\mathrm{j}=1}^{\mathrm{N}} \mathrm{h}_{\mathrm{xy} 1}(\mathrm{i}, \mathrm{j}) \\
& \mathrm{H}_{\mathrm{XY} 2}=-\sum_{\mathrm{i}=1}^{\mathrm{N}} \sum_{\mathrm{j}=1}^{\mathrm{N}} \mathrm{h}_{\mathrm{xy} 2}(\mathrm{i}, \mathrm{j}) \\
& \mathrm{F}_{\mathrm{INF} 1}=\frac{\mathrm{H}_{\mathrm{XY}}-\mathrm{H}_{\mathrm{XY} 1}}{\max \left(\mathrm{H}_{\mathrm{X}}, \mathrm{H}_{\mathrm{Y}}\right)} \\
& F_{I N F 2}=\sqrt{1-\exp \left\{-2\left(\mathrm{H}_{\mathrm{XY} 2}-\mathrm{H}_{\mathrm{XY}}\right)\right\}} \\
& F_{\text {IDM }}=\sum_{\mathrm{i}, \mathrm{j}} \frac{\mathrm{P}(\mathrm{i}, \mathrm{j})}{1+|\mathrm{i}-\mathrm{j}|}
\end{aligned}
$$


$\mathrm{F}_{\mathrm{ENG}}$ represents of the homogeneous energy measure of the image texture. This energy value is high for the homogeneous images. $\mathrm{F}_{\mathrm{CON}}$ is a measure of the texture contrast. This value is high for the high contrast images. $\mathrm{F}_{\mathrm{CORR}}$ represents the measure of correlation between the pixels located at the specific positions relative to each other. $\mathrm{F}_{\mathrm{VAR}}$ represents the measure of variance in the image texture. The variance value is high when the values deviate largely from the average value. $\mathrm{F}_{\mathrm{HOM}}$ is a measure of the homogeneity value of the image texture. $\mathrm{F}_{\mathrm{AVG}}$ is the sum average value proportional to the sum of the left-side diagonal elements of the GLCM. $\mathrm{F}_{\mathrm{SE}}$ is the sum entropy and is a measure of the randomness of the image. $F_{\mathrm{SV}}$ is the sum variance and is a measure of the deviation from the entropy. $\mathrm{F}_{\mathrm{ENT}}$ denotes the entropy of the image. The entropy value is high for the non-homogeneous image. $\mathrm{F}_{\mathrm{INF} 1}$ and $\mathrm{F}_{\mathrm{INF} 2}$ denote the information measures of correlation features 1 and 2. $F_{\mathrm{IDM}}$ denotes the Inverse Difference Moment of the image texture. For a highly textured image, the value of IDM is 0 , and the value is 1 for the untextured image. The curvelet transformation provides three mean values. Three values are obtained from the ATP extraction process. The Tamura features output nine features and convoluted GLCM outputs 12 features. Totally, 24 features are obtained. In the feature extraction process, $1 * 24$ features are obtained for each patch of the image and totally $100 * 24$ features are obtained for the whole image. Figure.16 shows the GUI images for the feature extraction process.

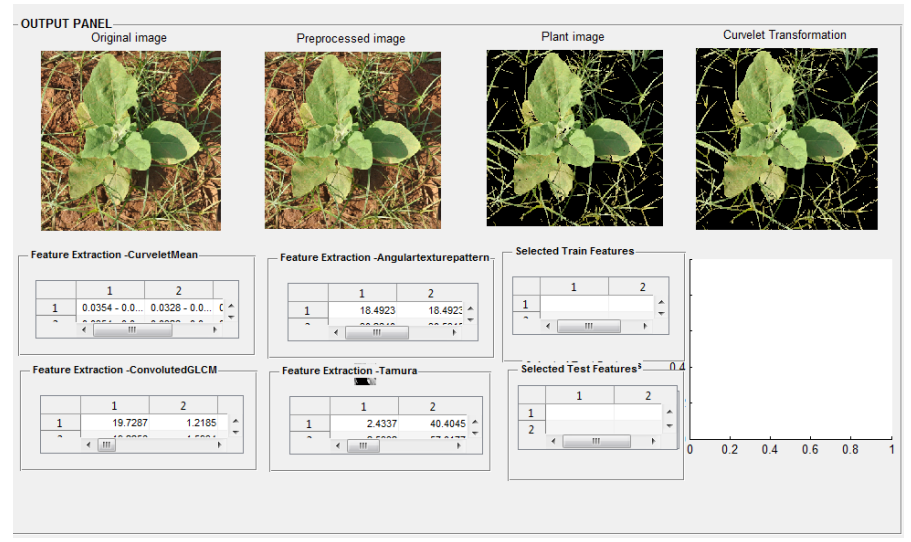

Figure 16: GUI images for feature extraction process

\section{Feature Selection and Classification}

The feature selection is performed by using PSO-combined Differential Evolution Feature Selection (DEFS) approach. This is done to select a minimum set of features to achieve better feature selection performance.

\subsection{PSO-Combined DEFS approach}

In the PSO technique, a population called as a swarm of candidate solutions is encoded as particles in the search space. PSO begins with the random initialization of the population. The whole swarm moves in the search space, for searching the best solution. Searching for the best solution is performed by updating the position of each particle. During the movement of the swarm, a vector $X_{i}=\left(X_{i 1}, X_{i 2}, \ldots, X_{i D}\right)$ represents the current position of the particle ' $\mathrm{i}$ '. $\mathrm{V}_{\mathrm{i}}=\left(\mathrm{V}_{\mathrm{i} 1}, \mathrm{~V}_{\mathrm{i} 2}, \ldots, \mathrm{V}_{\mathrm{iD}}\right)$ represents the velocity of the particle in the range of $\left[-\mathrm{v}_{\max }, \mathrm{v}_{\max }\right]$. The best previous position of a particle is denoted as personal best $\mathrm{P}_{\text {best }}$. The global best position obtained by the population is denoted as $\mathrm{G}_{\text {best }}$. The PSO searches for the optimal solution by updating the velocity and position of each particle, based on the $\mathrm{P}_{\text {best }}$ and $\mathrm{G}_{\text {best }}$. The PSO reduces 24 features to 20 features.

Differential Evolution (DE) is a parallel and direct search method that provides near-optimal solutions for the fitness function of the optimization problem. The parameters of the search space are encoded in the form of strings. A collection of the strings is termed as population denoted as ' $\mathrm{P}$ '. It is a collection of predefined number ' $\mathrm{N}$ ' of parameter vectors $x_{j, G}=\left[x_{1, j, G}, x_{2, j, G}, \ldots \ldots, x_{D, j, G}\right]$, where $\mathrm{j}=1,2, \ldots, \mathrm{N}$ for each generation ' $\mathrm{G}$ '. ' $\mathrm{D}$ ' represents the 
number of real parameters. The value of ' $\mathrm{N}$ ' does not change during the minimization process. Random selection of the initial vector population that represents the different points in the search space is performed. The fitness function is associated with each string that represents the degree of the wellness of the string. During the mutation operation, the weighted difference between the two population vectors is added to a third vector, to generate new parameter vectors. Then, the crossover operation is performed by mixing the mutated vector's parameters with the parameters of the target vector, to produce the trial vector. During the selection process, the target vector is replaced by the trial vector in the subsequent generation, if the trial vector produces a lower cost function value than the target vector. The process of selection, crossover and mutation continue for a fixed number of generations or until a termination condition is satisfied. The DEFS reduces 20 features to 15 features. Figure.17(a) and Figure.17 (b) show the PSO output for the images in dataset 1 and dataset 2. The PSO combined DEFS algorithm is described below in the following section.

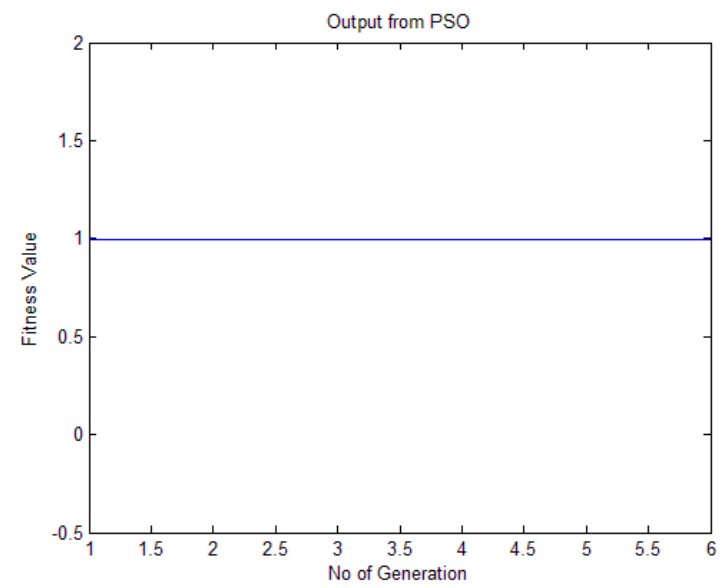

(a)

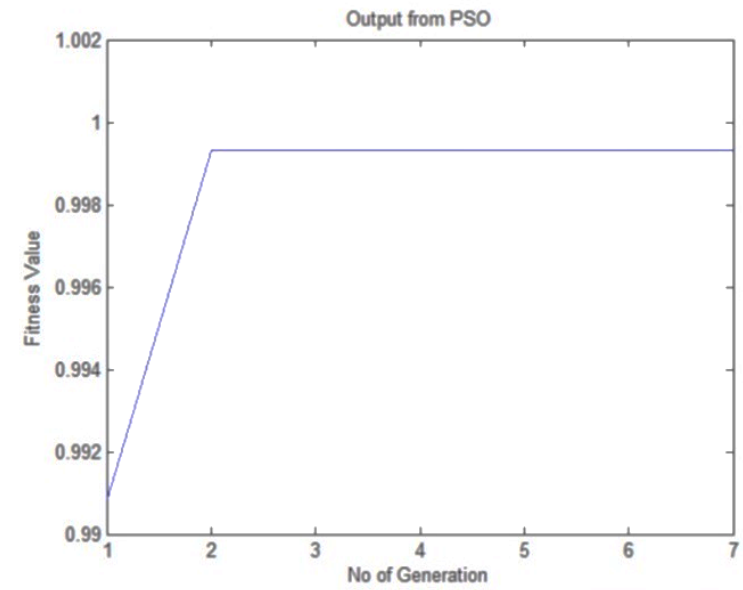

(b)

Figure 17: (a) and (b) PSO output plot for images in dataset 1 and dataset 2

\section{PSO combined DEFS Algorithm}

Input: Feature Values, Number of parameters to be optimized, maximum number of iteration

Step 1: Randomly initialize position, No of Iterations, Velocity of Each Particle

Step 2: While i< maxiteration

Step 3: Evaluate Fitness for each particle

Step 4: $\operatorname{Val}=\sum_{i=1}^{n} \operatorname{skewness}(i, j) / \operatorname{skewness}(i+1, j)$;

Step 5: pbest=val;

Step 6: For i=1 to popsize $\boldsymbol{d o}$

Step 7: $\quad$ update $\mathrm{P}_{\text {best }}$ of particle

Step 8: update $\mathrm{G}_{\text {best }}$ of particle

Step 9: $\quad$ For $\mathrm{i}=1$ to PopulationSize $d \boldsymbol{d o}$

Step 10: $\quad$ For $\mathrm{d}=1$ to Dimensionality $\boldsymbol{d o}$

Step 11: $\quad$ update the velocity of particle i

Step 12: $\quad$ update the position of particle i

Step 13: $\quad$ End For

Step 14: $\quad$ End For

Step 15: End For

Step 16: End While 
The fitness value for each particle is evaluated based on the skewness of the feature values. The personal best solution of the particle is determined from the fitness value. The personal best solution and global best solution of the particle are updated. Finally, the position and velocity of the particle are updated. After the selection of features, the classification of the image is performed by using the RVM-based classification. Figure.18 shows the GUI image for feature selection process.

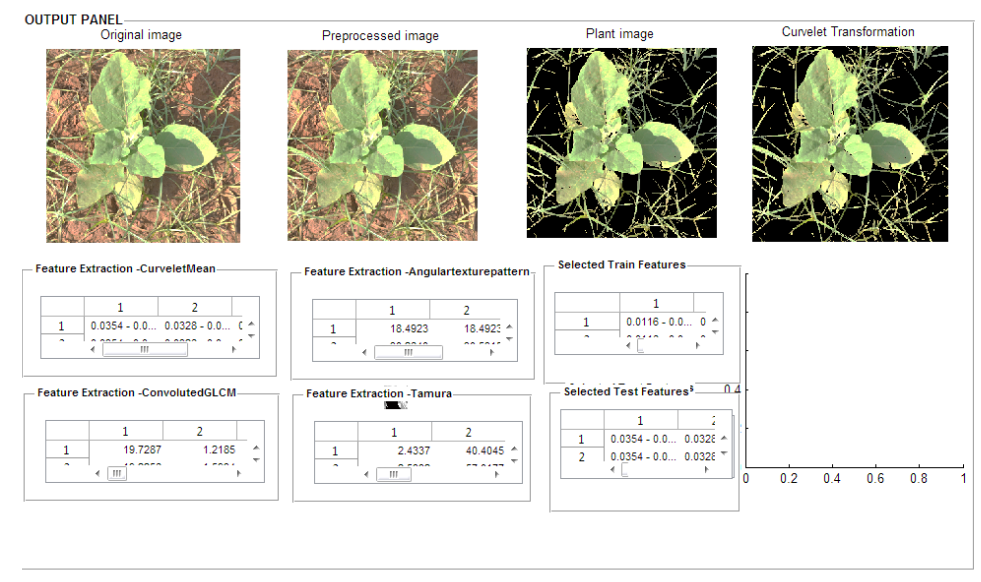

Figure 18: GUI Image for feature selection process

\subsection{RVM-based classification}

The classification of the selected features in the plant image is performed using the RVM-based classification technique. Selected features are learned and passed through the RVM classifier for finding the weed. RVM is a Bayesian regularization framework to obtain the solution for binary classification. The training time of the RVM shows the quadratic increase with the increase in the number of data points. The likelihood is defined as

$$
P(c / w)=\prod_{i=1}^{n} \sigma\left\{( y ( x _ { i } ) \} ^ { c _ { i } } \left[1-\sigma\left\{\left(y\left(x_{i}\right)\right\}\right]^{1-c_{i}}\right.\right.
$$

Where $\mathrm{X}=\left(\mathrm{x}_{1}, \mathrm{x}_{2}, \ldots \ldots, \mathrm{x}_{\mathrm{n}}\right)$ is the training data having class labels $\mathrm{C}=\left(\mathrm{c}_{1}, \mathrm{c}_{2}, \ldots \ldots, \mathrm{c}_{\mathrm{n}}\right)$ with $\mathrm{c}_{\mathrm{i}} \in(-1,1)$. $\sigma(y)$ is the logistic sigmoid function defined as

$$
\sigma(y(x))=\frac{1}{1+\exp (-y(x))}
$$

An iterative method is used to obtain $P(c / w)$. Let $\alpha_{i}^{*}$ denotes the maximum aposteriori estimate of the hyperparameter $\alpha_{i}$. The maximum aposteriori estimate of the weights $\left(W_{m}\right)$ is obtained by maximizing the following objective function

$$
f\left(w_{1}, w_{2}, \ldots, w_{n}\right)=\sum_{i=1}^{n} \log p\left(c_{i} / w_{i}\right)+\sum_{i=1}^{n} \log p\left(w_{i} / \alpha_{i}^{*}\right)
$$

In the resulting solution, the gradient of the objective function with respect to weight is calculated. The training data having non-zero coefficients $\mathrm{w}_{\mathrm{i}}$ called as relevance vectors contribute to the decision function. The RVM classification is performed to reduce the dimensionality of feature set and correct grouping of classified feature set vectors by using a small number of features. The crop region in the plant image is classified as 1 . After identifying the crop region, remaining blocks are extracted from the plant image. Figure.19 (a) and Figure.19 (b) show the RVM classified crop images of dataset 1 and dataset 2. Figure.20 (a) and Figure.20 (b) show the extraction of the 
remaining regions from the plant image, after identifying the crop blocks. Figure. 21 shows the GUI image for weed classification.

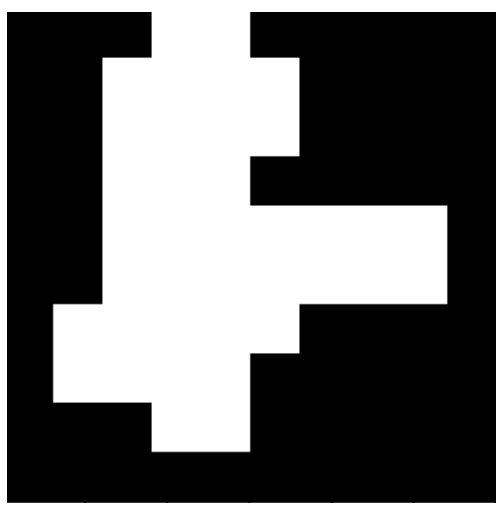

(a)

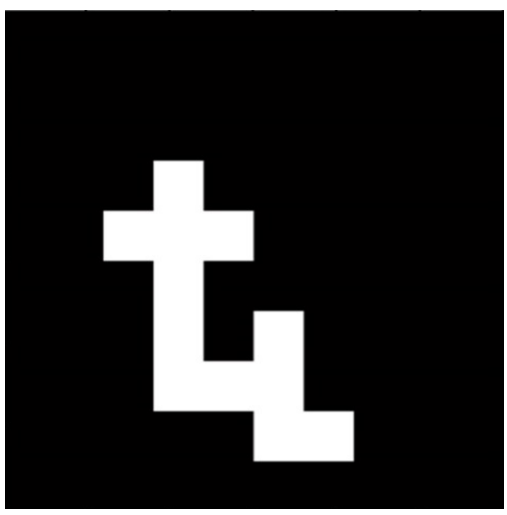

(b)

Figure 19: (a) and (b) RVM classified crop images of dataset 1 and dataset 2

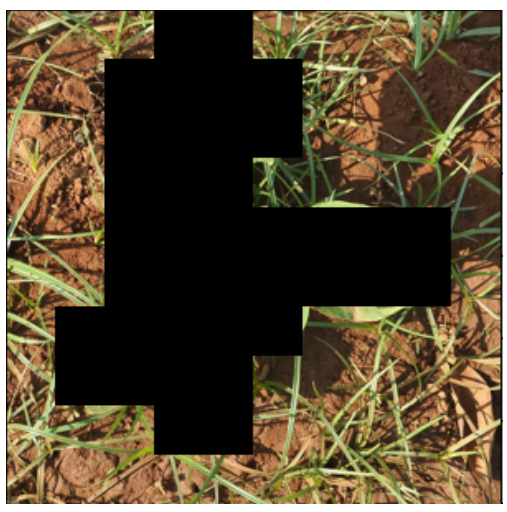

(a)

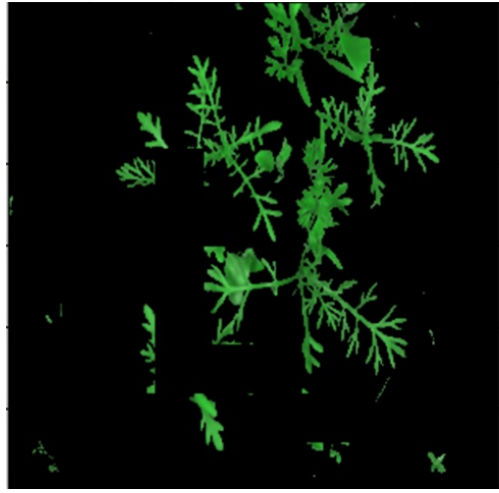

(b)

Figure 20: (a) and (b) Extraction of the remaining regions from the plant images of dataset 1 and dataset 2

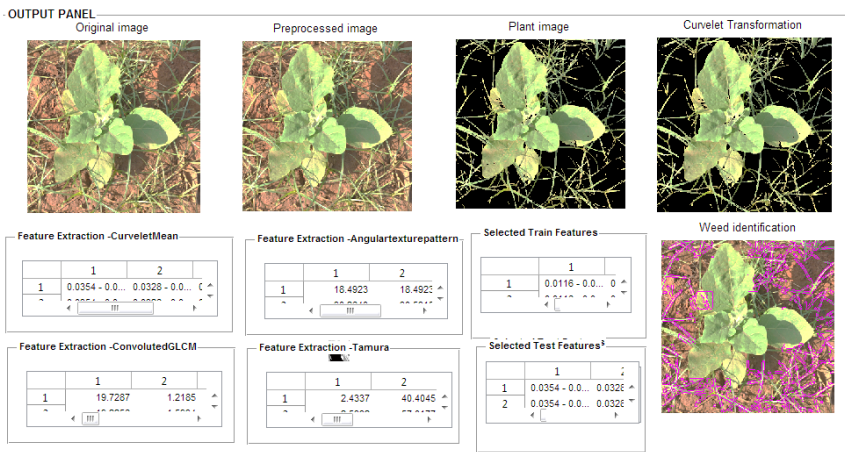

Figure 21: GUI image for weed classification 


\subsection{Weed Edge detection and Contouring}

The Canny-based edge detection method is used to detect the edges of the weeds from the image. Initially, the canny edge detector performs smoothing of the image to filter the noise in the original image, before locating and detecting the edges. The Gaussian filter is used in the Canny algorithm since it is computed using a simple mask. Once the suitable mask is decided, Gaussian smoothing is performed using the standard convolution techniques. The convolution mask is made to slide over the image, to manipulate the square of pixels. During the increase in the Gaussian Width, there is a slight increase in the localization error in the detected edges. After performing the image smoothing and noise removal, the edge strength is found out by considering the gradient value of the image. The Sobel operator performs the two-dimensional spatial gradient measurement. Then, the approximate absolute gradient magnitude at each point is found. The Sobel operator utilizes a pair of $3 \times 3$ convolution masks, to estimate the gradient along the $\mathrm{X}$-direction and $\mathrm{Y}$-direction. The magnitude of the gradient is approximated using the formula:

$$
|G|=\left|G_{x}\right|+\left|G_{y}\right|
$$

The edge direction is computed using the gradient values along the ' $\mathrm{X}$ ' and ' $\mathrm{Y}$ ' directions. If $\mathrm{G}_{\mathrm{x}}$ is equal to zero, the edge direction is equal to 0 or 90 degree, based on the value of $G_{y}$. If $G_{y}$ is equal to zero, the edge direction is equal to 0 degree. The edge direction is computed by using the following equation

$$
\theta=\tan ^{-1} \frac{\mathrm{G}_{y}}{\mathrm{G}_{\mathrm{x}}}
$$

Once the edge direction is computed, the edge direction is related to the tracing direction in the image. Then, non-maximum suppression is used to trace along the edges and suppress the pixel value that is not considered as edge pixels. A thin line is formed in the output image. Then, hysteresis thresholding is used for detecting the real edges using the maximum and minimum threshold values. The edges having the gradient intensity value more than the maximum threshold value are considered as the real edges. The gradient value of the edges lying below the minimum threshold value is detected as non-edges and discarded. The edges lying between the thresholds are classified as edges or non-edges based on their connectivity. Figure.22 (a) and Figure.22 (b) show the weed edge detection for dataset 1 and dataset 2 .

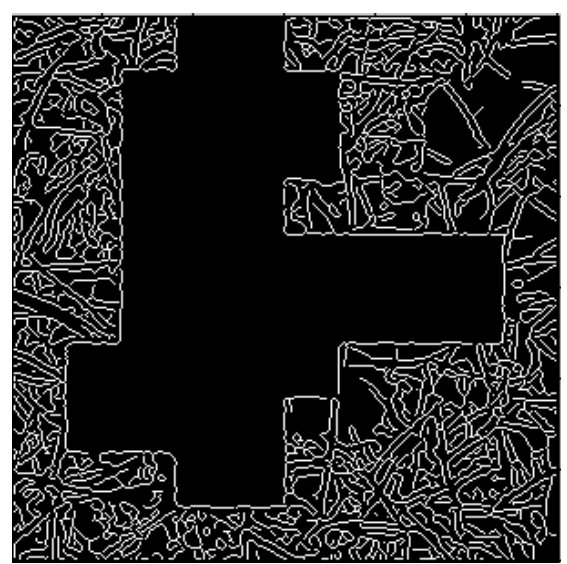

(a)

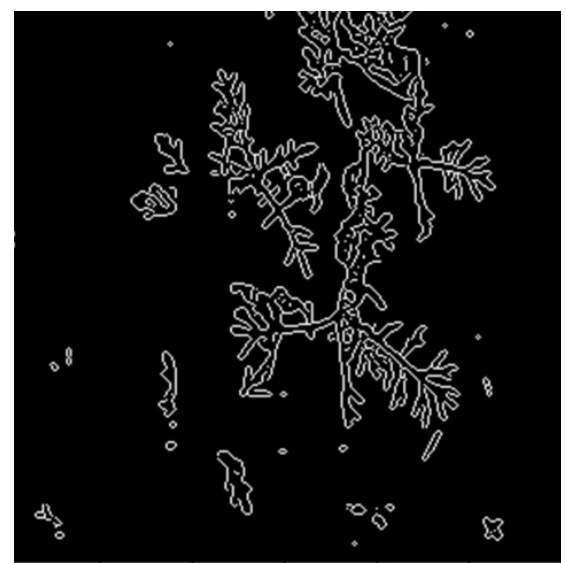

(b)

Figure 22: (a) and (b) Weed edge detection for dataset 1 and dataset 2 


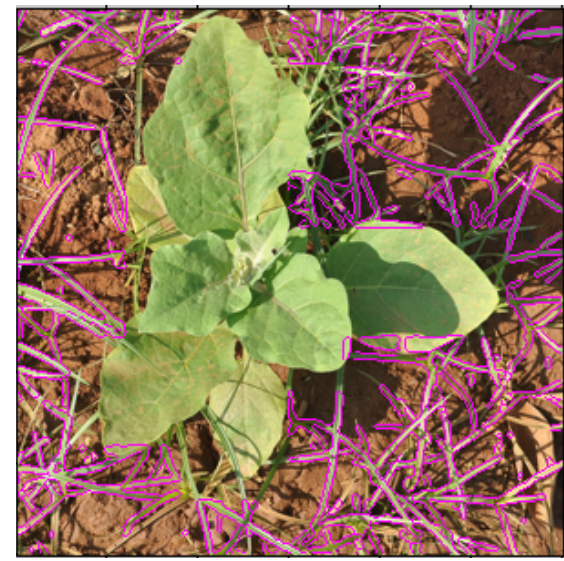

(a)

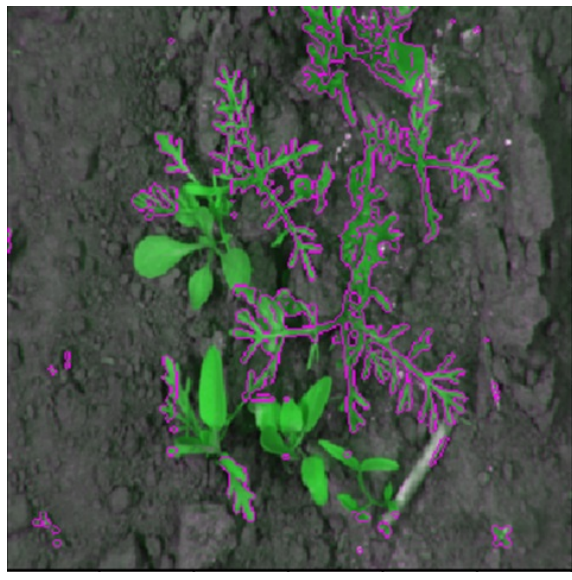

(b)

Figure 23: (a) and (b) Weed identification for dataset 1 and dataset 2

After detecting the edges, the contour is drawn over the edges. Then, the edge pixels are colored for the identification of weed in the plant image. Figure.23 (a) and Figure.23 (b) shows the weed identification for dataset 1 and dataset 2 .

\subsection{Fuzzy Rule-based Weed Patchiness Detection}

The Fuzzy rule-based approach is used for detecting the weed patchiness level [23]. The Fuzzy Logic Toolbox of MATLAB is used to develop the fuzzy logic algorithm. Fuzzy logic control is based on the mathematical concept called as "membership function". The weed patchiness is applied as an input to the toolbox and the level of the weed patchiness is obtained as the output. There are three levels of the weed pixels are high, medium and low. If the weed patchiness lies within the specific range of the weed patchiness, it is classified as 'medium' and 'low' levels. Otherwise, it is considered as 'high' level. Figure.24 shows the Fuzzy toolbox.

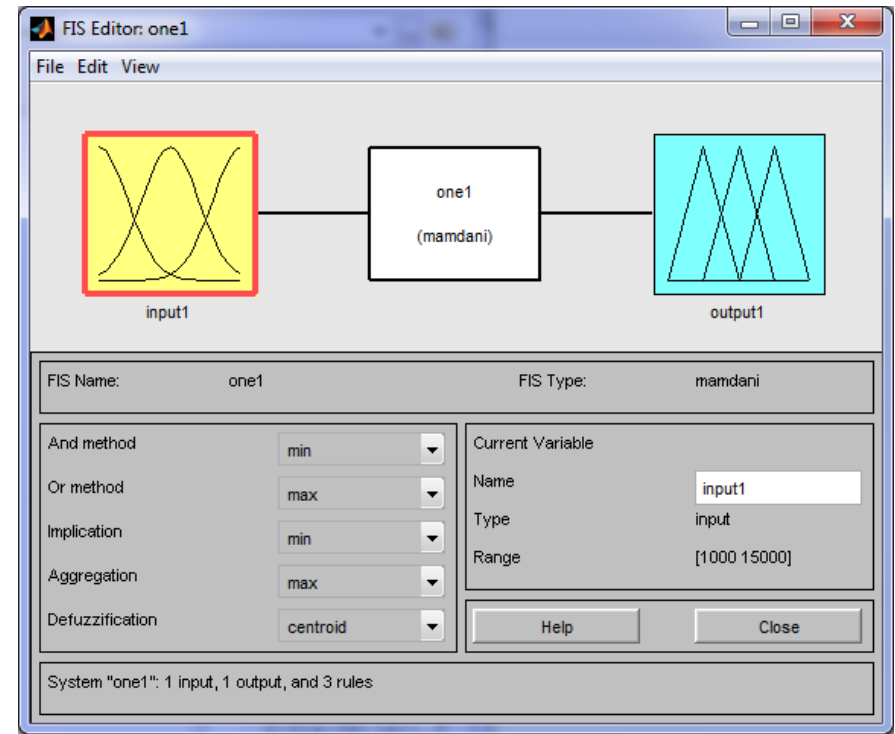

Figure 24: Fuzzy Toolbox 


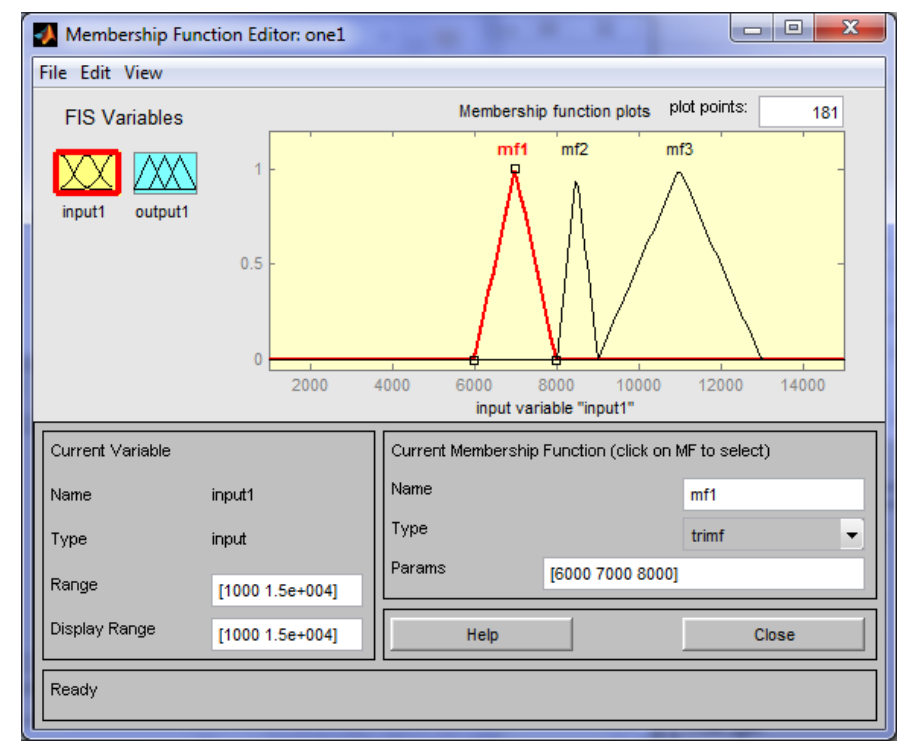

Figure 25: Fuzzy Logic Model including Membership function plots

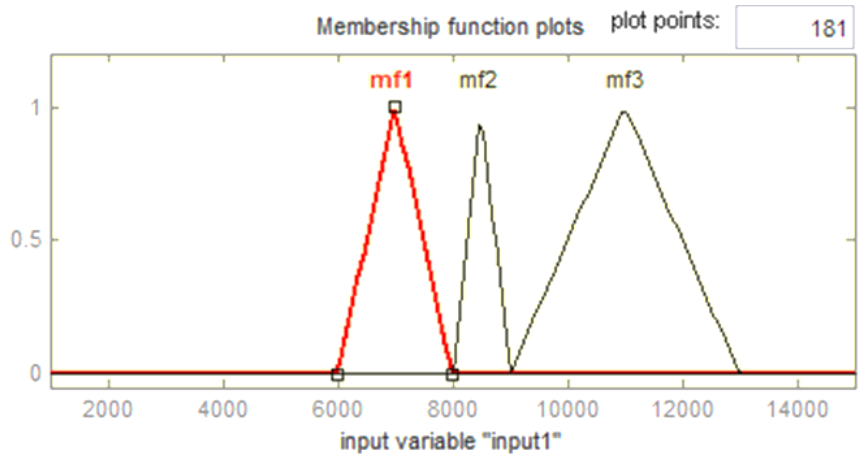

Figure 26: Membership function plots

Figure.25 shows the Fuzzy logic model including the membership function plots. Figure.26 shows the membership function plots. If the weed patchiness value lies between 6000 and 8000, it is considered as 'Low' level. If the patchiness lies between 8000 and 9000, it is considered as 'Medium' level. If the patchiness lies beyond 9000, it is considered as 'high' level.

\section{Performance Analysis}

This section presents the performance analysis results of the proposed ATP extraction method. In the proposed work, the angular texture pattern of the image is extracted, and selection of the optimal features is performed using the PSO-based DEFS approach. Classification of the crop region is done using RVM-based approach. With the extraction of the angular texture pattern at each windowing patch, clear analysis of the plant image in the complex background is achieved. In our work, Dataset 1 includes the Real-time plant image obtained from the Agricultural College and Research Institute, Madurai. Dataset 1 includes 500 images. The dataset 2 is a benchmark dataset comprising 60 images for crop/weed discrimination [26]. This dataset includes the field images in the top-down view acquired using the autonomous field robot in an organic carrot farm. Totally 560 images are used in our proposed work. $70 \%$ of the images are used for training and $30 \%$ are used for testing. A set of 410 images is used for training, and a set of 150 images is used for testing. Totally, 560 images are considered for evaluating the 
performance of the proposed approach. The metrics used for evaluating the performance of the proposed approach are

- Jaccard Distance

- Dice Distance

- Sensitivity

- Specificity

- Accuracy

- Hausdorff Distance

The accuracy level of the proposed method is compared with the accuracy levels of the existing techniques such as SVM approach for classification of crops and weeds from digital image [10] and SVM based Crop/weed classification in maize fields [27].

\subsection{Jaccard/Dice Similarity Measures}

Jaccard distance is defined as the measure of non-similarity between the images ' $\mathrm{A}$ ' and ' $\mathrm{B}$ '. It is written as the ratio of the common features to the total number of features. This is given as

$$
J_{d}(A, B)=1-J(A, B)=\frac{|A \cap B|}{|A \cup B|}
$$

Dice distance is defined as the measure of the degree of spatial overlap between the two similarities labeled regions over the average volume of these regions.

$$
D(A, B)=\frac{2 A B}{|A|+|B|}
$$

Figure.27 shows the Jaccard/Dice Similarity Measures of the proposed approach. From the graph, it is clearly observed that the Jaccard distance of the proposed approach is found to be low, and Dice distance is found to be higher. This indicates the better similarity between the images.

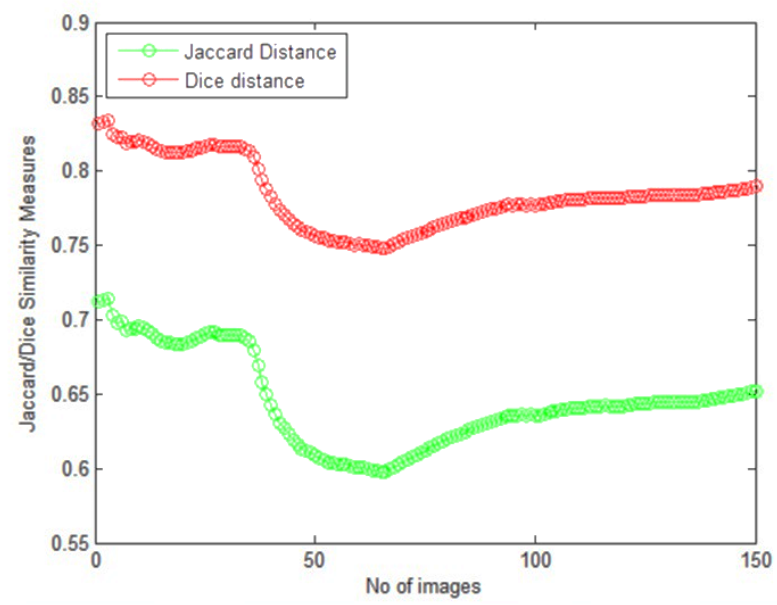

Figure 27: Jaccard/Dice Similarity Measures 


\subsection{Accuracy, Sensitivity, and Specificity}

Accuracy is defined as the measure of the correctly classification results of the weeds in the plant image. Sensitivity and Specificity are the most important measures to evaluate the performance of the RVM classifier. RVM classifier shows higher sensitivity and specificity value for the input plant images. Sensitivity is a true positive measure indicating the correct classification rate of the weeds. Specificity is a measure of true negative classifications that denotes the incorrect classification of the weeds.

$$
\begin{gathered}
\text { Accuracy }=\frac{\mathrm{N}_{\mathrm{TP}}+\mathrm{N}_{\mathrm{TN}}}{\mathrm{N}_{\mathrm{TP}}+\mathrm{N}_{\mathrm{TN}}+\mathrm{N}_{\mathrm{FP}}+\mathrm{N}_{\mathrm{FN}}} \\
\text { Sensitivity }=\frac{\mathrm{N}_{\mathrm{TP}}}{\mathrm{N}_{\mathrm{TP}}+\mathrm{N}_{\mathrm{FN}}} \\
\text { Specificity }=\frac{\mathrm{N}_{\mathrm{TN}}}{\mathrm{N}_{\mathrm{TN}}+\mathrm{N}_{\mathrm{FP}}}
\end{gathered}
$$

Where $\mathrm{N}_{T P}$ is the true positive measurement, $\mathrm{N}_{\mathrm{TN}}$ is the true negative measurement, $\mathrm{N}_{\mathrm{FP}}$ is the false positive measurement (a portion of the image incorrectly classified as weed), and $\mathrm{N}_{\mathrm{FN}}$ is the false negative measurement (a portion of the image incorrectly classified as not a weed).

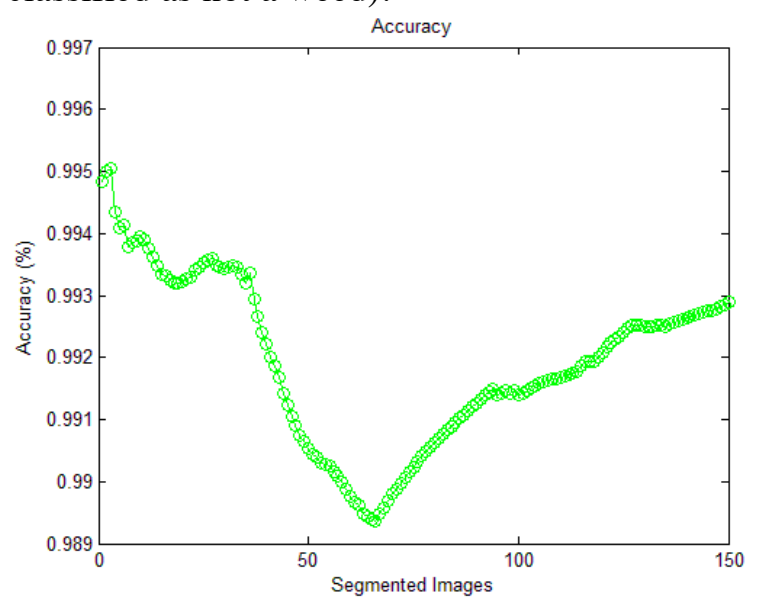

Figure 28: Accuracy of weed classification process

Figure. 28 shows the accuracy of the classification result. The proposed approach achieves better performance in terms of the weed classification accuracy.

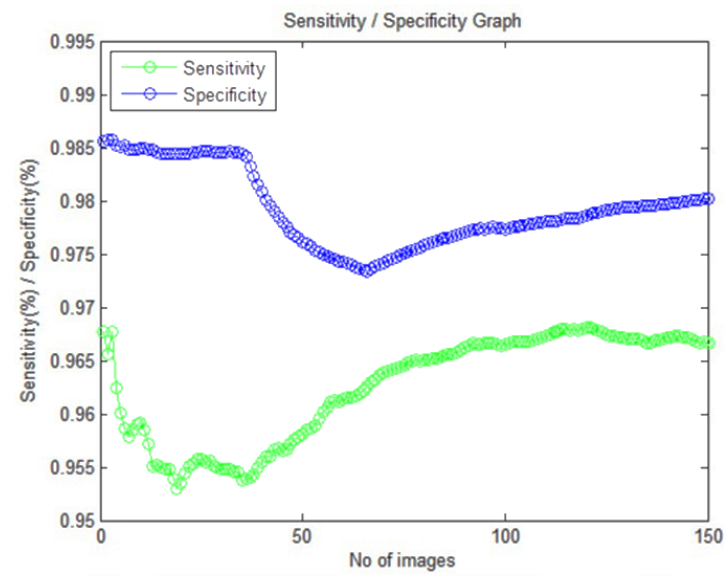

Figure 29: Sensitivity/Specificity of weed classification process 
Figure.29 shows the sensitivity and specificity of the weed classification process. The proposed approach achieves high sensitivity and specificity.

\subsection{Hausdorff distance}

The Hausdorff distance or Hausdorff metric is a measure of the degree of mismatch between the images. It is defined as

$$
h(A, B)=\max \{\operatorname{dist}(A, B), \operatorname{dist}(B, A)\}
$$

Figure.30 shows the Hausdorff distance metric for the proposed approach. The Hausdorff distance remains constant for most of the images. This implies the higher classification accuracy of the proposed approach.

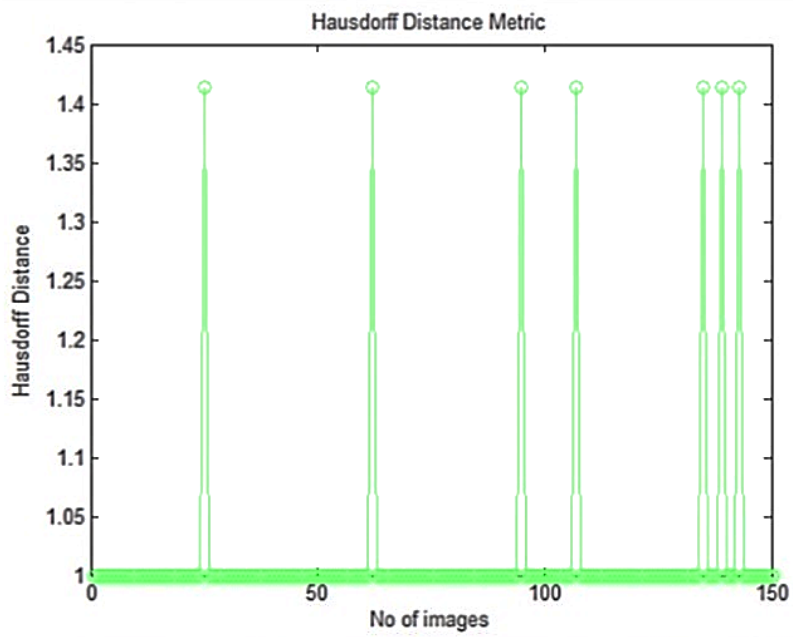

Figure 30: Hausdorff Distance

Figure.31 shows the GUI image for performance evaluation including the time-based evaluation of the Hausdorff distance, Jaccard Distance, Dice Distance, Accuracy, Sensitivity and Specificity of the proposed approach. The average accuracy of the proposed approach is $98.3756 \%$, average sensitivity is $99.4963 \%$ and specificity is $82.1588 \%$. The Jaccard distance is $98.2844 \%$ and Dice distance is $99.1348 \%$.

Table 1: Confusion Matrix

\begin{tabular}{|c|c|c|c|}
\hline \multicolumn{2}{|c|}{ Confusion Matrix } & \multicolumn{2}{c|}{ Predicted Class } \\
\hline \multirow{3}{*}{ Actual Class } & & Class 1 & Class 2 \\
\cline { 2 - 4 } & Class 1 & 83758 & 1038 \\
\cline { 2 - 4 } & Class 2 & 424 & 4780 \\
\hline
\end{tabular}




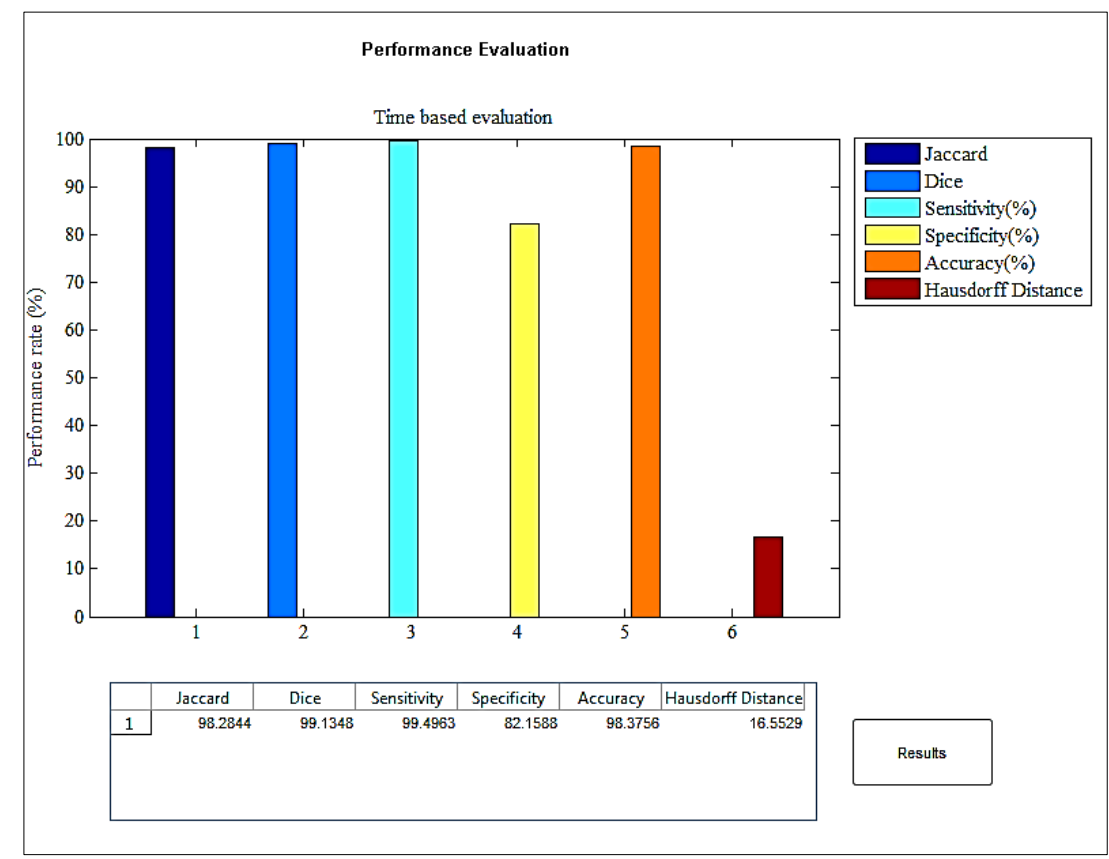

Figure 31: GUI image for performance evaluation

Table 1 shows the confusion matrix. The True Positive (TP) is 83758, False Negative (FN) is 1038, False Positive (FP) is 424 and True Negative (TN) is 4780 . Higher TP values and lower FP values indicate the efficiency of the proposed approach. Table 2 shows the comparative analysis of Hausdorff distance, Jaccard distance, Dice distance, Accuracy, Sensitivity and Specificity of the proposed approach for 5 images. The proposed approach achieves superior performance in terms of Hausdorff distance, Jaccard distance, dice distance, accuracy, sensitivity and specificity.

Table 2: Comparative analysis of Hausdorff distance, Jaccard distance, Dice distance, Accuracy, Sensitivity and Specificity of the proposed approach

\begin{tabular}{|c|c|c|c|c|c|c|}
\hline $\begin{array}{c}\text { Number } \\
\text { of } \\
\text { Images }\end{array}$ & $\begin{array}{c}\text { Hausdorff } \\
\text { distance }\end{array}$ & Jaccard & Dice & Accuracy (\%) & Sensitivity (\%) & Specificity (\%) \\
\hline 1 & 1 & 97.0605 & 98.5083 & 99.48 & 99.5619 & 99.4629 \\
\hline 2 & 1 & 92.7232 & 96.2242 & 99.5000 & 98.5562 & 99.5652 \\
\hline 3 & 1 & 98.3394 & 99.1628 & 99.5100 & 99.6718 & 99.4436 \\
\hline 4 & 1 & 90.7048 & 95.1259 & 99.4300 & 97.8308 & 99.5264 \\
\hline 5 & 1 & 89.2467 & 94.3178 & 99.4100 & 97.8681 & 99.4912 \\
\hline
\end{tabular}


Table 3: Comparative analysis of accuracy of the proposed and existing techniques

\begin{tabular}{|l|l|}
\hline Methods & $\begin{array}{l}\text { Accuracy } \\
(\%)\end{array}$ \\
\hline SVM based Crop/weed classification in maize fields & 93.1 \\
\hline $\begin{array}{l}\text { SVM approach for classification of crops and weeds from } \\
\text { digital image }\end{array}$ & 97.3 \\
\hline Proposed ATP Extraction Method & 99.3 \\
\hline
\end{tabular}

Table 3 shows the comparative analysis of the accuracy of the proposed ATP extraction method and existing techniques. The proposed method is compared with the existing SVM approach for classification of crops and weeds from the digital image [10] and SVM based Crop/weed classification in maize fields [27]. The overall accuracy of the proposed approach is $99.3 \%$, which is relatively higher than the existing techniques. Hence, the proposed approach is found to be efficient than the existing techniques.

Table 4: Classification accuracy of patches analyzed in 2008 Quick-Bird image using different classification algorithms

\begin{tabular}{|c|c|c|c|c|c|c|c|c|}
\hline \multirow{2}{*}{$\begin{array}{c}\text { Field } \\
\text { Number }\end{array}$} & \multicolumn{2}{|c|}{ Parallelepiped } & \multicolumn{2}{c|}{ Mahalanobis Distance } & \multicolumn{2}{c|}{ Maximum Likelihood } & \multicolumn{2}{c|}{ Proposed CTATP } \\
\cline { 2 - 8 } & OA (\%) & Kappa & OA (\%) & Kappa & OA (\%) & Kappa & OA (\%) & Kappa \\
\hline 1 & 37.4 & 0.1 & 95.4 & 0.9 & 97.6 & 1 & 98.17 & 1 \\
\hline 2 & 91.5 & 0.8 & 99.7 & 1 & 99.8 & 1 & 99.83 & 1 \\
\hline 3 & 68.3 & 0.5 & 98.4 & 1 & 98.1 & 1 & 98.42 & 1 \\
\hline 4 & 89.7 & 0.8 & 100 & 1 & 100 & 1 & 100 & 1 \\
\hline 5 & 59.2 & 0.4 & 99.8 & 1 & 100 & 1 & 100 & 1 \\
\hline
\end{tabular}

The Quick-Bird (QB) images are obtained in the wheat field located in Spain, on May 2006 and 2008. Naturally, the wheat fields in the study area are infested with wild oat. The wheat crops are at the initial senescence stage and the wild oat weed patches are at the advanced seed maturation stage. The images are obtained with a spatial resolution of $2.4 \mathrm{~m}$ and a radiometric resolution of 11 bits during spring season in the experimental conditions [15].

Table 4 shows the classification accuracy of the patches analyzed in 2008 QB image using different classification algorithms. The Overall Accuracy (OA) and Kappa of the parallelepiped, Mahalanobis Distance, Maximum Likelihood and proposed CTATP approach. The wild oat patches present in the infested wheat fields is discriminated accurately by the proposed CTATP approach. The OA and Kappa of the proposed approach are higher than the parallelepiped, Mahalanobis Distance, Maximum Likelihood. Table 5 illustrates the classification accuracy of wheat fields obtained with the first-level segmentation using Mahalanobis Distance, Maximum Likelihood algorithms in 2006 and 2008 QB imagery and Proposed CTATP approach. The classification accuracy of the Mahalanobis Distance and Maximum Likelihood algorithms is greater than $85 \%$. But, the OA accuracy of the proposed CTATP approach is greater than 95\% and Kappa value is greater than 0.9 for the 2006 and 2008 QB image. 
Table 5: Classification accuracy of wheat fields obtained with the first-level segmentation using Mahalanobis

Distance, Maximum Likelihood algorithms in 2006 and 2008 QB imagery and Proposed CTATP approach

\begin{tabular}{|c|c|c|c|c|c|c|}
\hline \multirow{2}{*}{$\begin{array}{c}\text { Quick- } \\
\text { Bird } \\
\text { Database }\end{array}$} & \multicolumn{2}{|c|}{ Mahalanobis Distance } & \multicolumn{2}{|c|}{ Maximum Likelihood } & \multicolumn{2}{c|}{ Proposed CTATP } \\
\cline { 2 - 7 } & OA (\%) & Kappa & OA (\%) & Kappa & OA (\%) & Kappa \\
\hline 2006 & 86.26 & 0.73 & 88.79 & 0.77 & 96.42 & 0.91 \\
\hline 2008 & 85.68 & 0.71 & 89.22 & 0.76 & 97.36 & 0.93 \\
\hline
\end{tabular}

The accuracy is defined as

$$
A C C=\left(\frac{1}{N}\right) \sum_{n=1}^{N}\left(I\left(y_{n}^{*}=y_{n}\right)\right)
$$

Where $I(\cdot)$ is the zero-one loss function, $\mathrm{N}$ is the total number of patterns in the dataset, $y_{n}$ is the desired output for pattern ' $\mathrm{n}$ ' and $y_{n}^{*}$ is the prediction.

The minimum sensitivity is defined as the minimum value of the sensitivities for each class:

$$
M S=\min \left\{S_{i} ; i=0, \ldots \ldots K\right\}
$$

Where $S_{i}$ is the sensitivity for the $\mathrm{i}^{\text {th }}$ class. Sensitivity for the class ' $\mathrm{i}$ ' corresponds to the correct classification rate for the specific class.

A set of aerial images are obtained from a sunflower field situated at the private farm in Spain, and in a maize field situated in the Spain. The crops were at the stage of 4-6 leaves unfolded. The fields were naturally infested by weeds of similar size or smaller size than the crop plants. The sunflower field is infested by pigweed, mustard and bindweed and the maize field is infested by saltwort. The experimental plot of $100 \times 100 \mathrm{~m}$ is delimited within the crop-fields to perform the flights. The coordinates of each corner of the flight area are collected using a Global Positioning System (GPS) to prepare the flight route in the mission-planning task. The experiments are conducted using the sunflower field and validated using the maize case. The accuracy and MS of the proposed CTATP approach are higher than the Random Selection method and Unsupervised Pattern Selection (UPS) method [28]. Table 6 depicts the mean results obtained for the Random Selection Method, UPS method and proposed CTATP approach.

Table 6: Mean results obtained for the Random Selection Method, UPS method and proposed CTATP approach

\begin{tabular}{|c|c|c|c|c|c|c|}
\hline \multirow{2}{*}{$\begin{array}{c}\text { Training } \\
\text { Data (\%) }\end{array}$} & \multicolumn{2}{|c|}{ Random Selection Method } & \multicolumn{2}{c|}{ UPS method } & \multicolumn{2}{c|}{ Proposed CTATP } \\
\cline { 2 - 7 } & Accuracy (\%) & MS & Accuracy (\%) & MS & Accuracy (\%) & MS \\
\hline $50 \%$ & $77.5 \pm 5.34$ & $58.0 \pm 11.35$ & $89.67 \pm 4.07$ & $77.5 \pm 9.2$ & $93.6 \pm 2.13$ & $87.5 \pm 1.39$ \\
\hline $75 \%$ & $76.17 \pm 5.83$ & $52.0 \pm 17.19$ & $90.67 \pm 2.38$ & $75.5 \pm 8.64$ & $96.4 \pm 2.83$ & $86.27 \pm 2.84$ \\
\hline
\end{tabular}

\section{Conclusion and Future Work}

The conclusion and future scope of the proposed work are discussed in this section. A novel ATP extraction method for the crop and weed discrimination using curvelet transformation is proposed in this paper. For the preprocessing operation, AMF is used to filter the impulse noise from the plant image and perform smoothening of the image for the clear analysis of textures in the image. Green pixel extraction is performed to obtain the green pixel count from the filtered image. K-means clustering is applied to the filtered image, to cluster the soil and plants. Then, the green pixel count is verified with the pixel count of the clusters. If the green pixel count is nearest 
to the pixel count of the clusters, then it is considered as the plant image. Curvelet transformation is applied to the plant image. After applying the curvelet transformation, the angular based features are extracted from the transformed image. Feature extraction is performed to extract the angular texture pattern of the plant image. The Tamura features are extracted from the image, Gabor filter with four orientations is applied to the image and GLCM features are extracted from the Gabor filtered image. PSO-based DEFS is applied to select the optimal features. Then, the selected features are classified using an RVM-based classifier to find out the weed. Cannybased Edge detection and contouring is performed based on the classified to identify the weed. The fuzzy rulebased approach is used for detecting the low, medium and high levels of the weed patchiness. Clear analysis and segmentation of the plant image in the complex background are achieved due to the angular texture pattern extraction at each windowing patch. The proposed approach achieves better performance in terms of Hausdorff distance, Jaccard distance, Dice Distance, Accuracy, Sensitivity, and Specificity. The accuracy of the proposed approach is higher than the existing SVM-based approaches. In our future work, the application amount of the herbicides in the weeded area is determined based on the Fuzzy-rule-based weed patchiness estimation result.

\section{References}

[1] A. Tellaeche, G. Pajares, X. P. Burgos-Artizzu, and A. Ribeiro, "A computer vision approach for weeds identification through Support Vector Machines," Applied Soft Computing, 11(1):908-915, 2011. doi:10.1016/j.asoc.2010.01.011

[2] J. Chaki, R. Parekh, and S. Bhattacharya, "Plant leaf recognition using texture and shape features with neural classifiers," Pattern Recognition Letters, 58:61-68, 2015. doi:10.1016/j.patrec.2015.02.010

[3] S. Haug, A. Michaels, P. Biber, and J. Ostermann, "Plant classification system for crop/weed discrimination without segmentation," in IEEE Winter Conference on Applications of Computer Vision (WACV), pp. 1142-1149, 2014. doi: 10.1109/WACV.2014.6835733

[4] F. Ahmed, M. H. Kabir, S. Bhuyan, H. Bari, and E. Hossain, "Automated weed classification with local pattern-based texture descriptors," Int. Arab J. Inf. Technol., 11:87-94, 2014.

[5] W. Kazmi, F. J. Garcia-Ruiz, J. Nielsen, J. Rasmussen, and H. J. Andersen, "Detecting creeping thistle in sugar beet fields using vegetation indices," Computers and Electronics in Agriculture, 112:10-19, 2015. doi:10.1016/j.compag.2015.01.008

[6] P. J. Herrera, J. Dorado, and Á. Ribeiro, "A new combined strategy for discrimination between types of weed," in ROBOT2013: First Iberian Robotics Conference, 469-480, 2014. doi:10.1007/978-3-31903413-3_34

[7] Z. Bo, W. H. Hua, L. S. Jun, M. W. Hua, and Z. X. Chao, "Research on weed recognition method based on invariant moments," in 11th World Congress on Intelligent Control and Automation (WCICA), 2014, pp. 2167-2169.doi: 10.1109/WCICA.2014.7053057

[8] N. M. Tahir, S. R. M. S. Baki, M. A. Hairuddin, and N. D. K. Ashar, "Classification of Elaeis Guineensis disease-leaf under uncontrolled illumination using RBF network," in IEEE International Conference on Control System, Computing and Engineering (ICCSCE), 2014, pp. 617-621.doi: 10.1109/ICCSCE.2014.7072792

[9] K. C. Swain, M. Nørremark, R. N. Jørgensen, H. S. Midtiby, and O. Green, "Weed identification using an automated active shape matching (AASM) technique," biosystems engineering, 110:450-457, 2011. doi:10.1016/j.biosystemseng.2011.09.011

[10] F. Ahmed, H. A. Al-Mamun, A. H. Bari, E. Hossain, and P. Kwan, "Classification of crops and weeds from digital images: A support vector machine approach," Crop Protection, 40:98-104, 2012. doi:10.1016/j.cropro.2012.04.024 
[11] T. Rumpf, C. Römer, M. Weis, M. Sökefeld, R. Gerhards, and L. Plümer, "Sequential support vector machine classification for small-grain weed species discrimination with special regard to Cirsium arvense and Galium aparine," Computers and electronics in agriculture, 80:89-96, 2012. doi:10.1016/j.compag.2011.10.018

[12] J. Torres-Sospedra and P. Nebot, "Two-stage procedure based on smoothed ensembles of neural networks applied to weed detection in orange groves," Biosystems Engineering, 123:40-55, 2014. doi:10.1016/j.biosystemseng.2014.05.005

[13] M. H. Siddiqi, S.-W. Lee, and A. M. Khan, "Weed Image Classification using Wavelet Transform, Stepwise Linear Discriminant Analysis, and Support Vector Machines for an Automatic Spray Control System," Journal of Information Science and Engineering, 30:1227-1244, 2014.

[14] H. Liu, S. H. Lee, and C. Saunders, "Development Of A Machine Vision System For Weed Detection During Both Of Off-Season And In-Season In Broadacre No-Tillage Cropping Lands," American Journal of Agricultural and Biological Sciences, 9:174-193, 2014. doi: 10.3844/ajabssp.2014.174.193

[15] I. L. Castillejo-González, J. M. Peña-Barragán, M. Jurado-Expósito, F. J. Mesas-Carrascosa, and F. López-Granados, "Evaluation of pixel-and object-based approaches for mapping wild oat (Avena sterilis) weed patches in wheat fields using QuickBird imagery for site-specific management," European Journal of Agronomy, 59:57-66, 2014.doi:10.1016/j.eja.2014.05.009

[16] J. T. Atkinson, R. Ismail, and M. Robertson, "Mapping bugweed (solanum mauritianum) infestations in pinus patula plantations using hyperspectral imagery and support vector machines," IEEE Journal of Selected Topics in Applied Earth Observations and Remote Sensing, 7:17-28, 2014. doi: 10.1109/JSTARS.2013.2257988

[17] F. J. Garcia-Ruiz, D. Wulfsohn, and J. Rasmussen, "Sugar beet (Beta vulgaris L.) and thistle (Cirsium arvensis L.) discrimination based on field spectral data," Biosystems Engineering, 139:1-15, 2015. doi: doi:10.1016/j.biosystemseng.2015.07.012

[18] M. Montalvo, J. M. Guerrero, J. Romeo, L. Emmi, M. Guijarro, and G. Pajares, "Automatic expert system for weeds/crops identification in images from maize fields," Expert Systems with Applications, 40:75-82, 2013. doi:10.1016/j.eswa.2012.07.034

[19] F.-M. De Rainville, A. Durand, F.-A. Fortin, K. Tanguy, X. Maldague, B. Panneton, et al., "Bayesian classification and unsupervised learning for isolating weeds in row crops," Pattern Analysis and Applications, 17:401-414, 2014.doi: 10.1007/s10044-012-0307-5

[20] A. Tannouche, K. Sbai, Y. Ounejjar, and A. Rahmani, "A Real Time Efficient Management of Onions Weeds Based on a Multilayer Perceptron Neural Networks Technique," International Journal of Farming and Allied Sciences, 4(2):161-166, 2015.

[21] A. M. Tobal and S. A. Mokhtar, "Weeds Identification Using Evolutionary Artificial Intelligence Algorithm," Journal of Computer Science, 10:1355-1361, 2014. doi: 10.3844/jcssp.2014.1355.1361

[22] M. Mursalin and M. Mesbah-Ul-Awal, "Towards Classification of Weeds through Digital Image," in Fourth International Conference on Advanced Computing \& Communication Technologies (ACCT), 2014, pp. 1-4.doi: 10.1109/ACCT.2014.101

[23] C.-C. Yang, S. O. Prasher, J.-A. Landry, and H. S. Ramaswamy, "Development of a herbicide application map using artificial neural networks and fuzzy logic," Agricultural Systems, 76:561-574, 2003. doi:10.1016/S0308-521X(01)00106-8

[24] S. H. Hlaing and A. S. Khaing, "Weed and crop segmentation and classification using area thresholding," IJRET, 3:375-382, 2014. 
[25] Y. Zhang, W. Gao, and J. Liu, "Integrating Color Vector Quantization and Curvelet Transform for Image Retrieval," Guest Editors, p. 99, 2011.

[26] S. Haug and J. Ostermann, "A Crop/Weed Field Image Dataset for the Evaluation of Computer Vision Based Precision Agriculture Tasks," in Computer Vision-ECCV 2014 Workshops, 2014, pp. 105-116.doi: 10.1007/978-3-319-16220-1_8

[27] J. M. Guerrero, G. Pajares, M. Montalvo, J. Romeo, and M. Guijarro, "Support vector machines for crop/weeds identification in maize fields," Expert Systems with Applications, 39:11149-11155, 2012. doi:10.1016/j.eswa.2012.03.040

[28] M. Pérez-Ortiz, J. M. Peña, P. A. Gutiérrez, J. Torres-Sánchez, C. Hervás-Martínez, and F. LópezGranados, "Selecting patterns and features for between-and within-crop-row weed mapping using UAVimagery," Expert Systems with Applications, 47:85-94, 2016. doi:10.1016/j.eswa.2015.10.043 